\title{
Associative Plasticity at Excitatory Synapses Facilitates Recruitment of Fast-Spiking Interneurons in the Dentate Gyrus
}

\author{
Sivakumar Sambandan, ${ }^{1,2}$ Jonas-Frederic Sauer, ${ }^{1,2}$ Imre Vida, ${ }^{3}$ and Marlene Bartos ${ }^{1,2}$ \\ ${ }^{1}$ Institute of Medical Sciences, University of Aberdeen, Foresterhill, Aberdeen AB25 2ZD, United Kingdom, ${ }^{2}$ Institut für Physiologie I, Universität Freiburg, \\ D-79108 Freiburg, Germany, and ${ }^{3}$ School of Life Sciences, College of Medicine, Veterinary Medicine, and Life Sciences, University of Glasgow, Glasgow G12 \\ $8 Q Q$, United Kingdom
}

\begin{abstract}
Fast-spiking perisomatic-inhibitory interneurons (PIIs) receive convergent excitation and mediate both feedforward and feedback inhibition in cortical microcircuits. However, it remains poorly understood how convergent excitatory inputs recruit PIIs to produce precisely timed inhibition. Here, we analyzed the interaction of inputs from the entorhinal cortex [perforant path (PP)] and from local granule cells [mossy fibers (MFs)] onto PIIs in the rat dentate gyrus (DG). PP stimulation alone activates PIIs with low temporal precision. Interestingly, when PP and MFs are coactivated with a $10 \mathrm{~ms}$ delay, PIIs discharge with precise timing. Moreover, repeated coactivation of the two inputs induces associative long-term potentiation (LTP) at MF synapses. Under these conditions, a single potentiated MF input is sufficient to recruit PIIs in a reliable and highly precise manner to provide feedback inhibition. MF-LTP depends on the discharge of PIIs, indicating Hebbian plasticity. However, MF-LTP is preserved when NMDA receptors are blocked but depends on transmission through $\mathrm{Ca}^{2+}$-permeable AMPA receptors (AMPARs). PP-PII synapses, in contrast, lack $\mathrm{Ca}^{2+}$-permeable AMPARs and do not show plasticity on associative activation. Thus, precise recruitment of PIIs requires excitation through MF-PII synapses during feedforward activation. We propose that associative plasticity at these synapses is a central mechanism that adjusts inhibition levels to maintain sparse activity and to improve signal-to-noise ratio in the DG network.
\end{abstract}

\section{Introduction}

The dentate gyrus (DG) acts as an interface between the neocortex and the hippocampus (Anderson et al., 1971). The main computational function of the DG is thought to be the separation of patterns from the rich neocortical input and to produce a sparse code for the CA3 area (Treves and Rolls, 1994; Leutgeb et al., 2007; McHugh et al., 2007). Consistent with this theory, DG granule cells (GCs) show a sparse activity pattern (Leutgeb et al., 2007), which seems to be governed by powerful GABAergic inhibition (Nitz and McNaughton, 2004).

Fast-spiking perisomatic-inhibitory interneurons (PIIs), such as basket cells, mediate rapid and precisely timed inhibition and therefore play a particularly important role in controlling the discharge of GCs (Jonas et al., 2004). Recruitment of PIIs, in turn, depends on the properties and timing of excitatory inputs (Geiger et al., 1997). In the DG, PIIs receive direct monosynaptic excita-

Received April 20, 2010; revised May 30, 2010; accepted July 18, 2010.

This work was supported by the Royal Society (M.B.), the Northern Research Partnership (J.-F.S.), the University of Aberdeen (S.S.), German Research Foundation Grant DFG SFB 505 TP-C6, and the Volkswagen Foundation (Lichtenberg Professorship; M.B.). We thank Drs. K. Lamsa, W. Wisden, and P. Wulff for critical comments on previous versions of this manuscript. We thank M. Strüber for constructive comments to this manuscript. We further thank K. Winterhalter and S. Becherer for technical assistance.

The authors declare no competing financial interests.

Correspondence should be addressed to Dr. Marlene Bartos at the above address. E-mail: marlene.bartos@ physiologie.uni-freiburg.de.

DOI:10.1523/JNEUROSCI.2012-10.2010

Copyright $\odot 2010$ the authors $\quad 0270-6474 / 10 / 3011826-12 \$ 15.00 / 0$ tion from perforant path (PP) afferents to their apical dendrites (Zipp et al., 1989) and indirect disynaptic excitation (PP-GCPII) via the axons of local GCs, the mossy fibers (MFs), to their basal dendrites (Geiger et al., 1997; Acsády et al., 1998). However, properties of these inputs seem to be partially incompatible with the proposed rapid and efficient recruitment of inhibition (Jonas et al., 2004). PP-PII synapses have a large electrotonic distance; therefore, efficiency and precision of PII activation is likely to be low (Nörenberg et al., 2010). MF-PII synapses, in contrast, are located more proximally and elicit a rapid postsynaptic conductance change (Geiger et al., 1997). Accordingly, MF inputs can discharge PIIs with high temporal precision, but a relatively large number of synchronously active MFs (5-20) is needed to reach action potential threshold (Geiger et al., 1997). This requirement is inconsistent with the sparse activity pattern of GCs observed in vivo (Jung and McNaughton, 1993; Leutgeb et al., 2007).

How can these discrepancies be reconciled? First, in intact networks, these excitatory inputs do not act in isolation, but rather converge onto PIIs within a narrow time window defined by the latencies at the monosynaptic (PP-PII) and disynaptic pathways (PP-GC-PII). Thus, during physiological activity patterns, $\mathrm{PP}$ input and delayed input from a low number of activated GCs (Jung and McNaughton, 1993) may act synergistically and recruit PIIs in a reliable and precise manner. Second, plastic changes at these input synapses, either by associative Hebbian (Hebb, 1949; Alle et al., 2001) or anti-Hebbian mechanisms (Lamsa et al., 2007) may increase synaptic strength of excitatory 
synapses and thereby enhance activation of the interneurons in a dynamic fashion. Indeed, in principal cells, distal excitatory inputs have been found to influence synaptic efficacy of proximal inputs (Buzsáki, 2002; Judge and Hasselmo, 2004; Dudman et al., 2007; Takahashi and Magee, 2009). If similar mechanisms apply to PIIs, the PP can act to control the gain of recurrent excitatory synapses and thereby the strength of feedback inhibition in the DG network.

Here, we tested these hypotheses by performing whole-cell and perforated-patch recordings and analyzing the functional impact of converging PP and MF inputs onto the activation and synaptic plasticity in fast-spiking PIIs.

\section{Materials and Methods}

\section{Hippocampal slice preparation}

Transverse 300- $\mu \mathrm{m}$-thick hippocampal slices were cut from 17- to 24-dold Wistar rats using vibratomes (Dosaka DTK-1000; Leica VT1200). Animals were killed according to the United Kingdom Animals (Scientific Procedures) Act (1986) and institutional guidelines. For dissection and slicing, a sucrose-based solution containing $87 \mathrm{~mm} \mathrm{NaCl}, 25 \mathrm{~mm}$ $\mathrm{NaHCO}_{3}, 2.5 \mathrm{~mm} \mathrm{KCl}, 1.25 \mathrm{~mm} \mathrm{NaH}_{2} \mathrm{PO}_{4}, 10 \mathrm{~mm}$ glucose, $0.5 \mathrm{~mm} \mathrm{CaCl}$, $7 \mathrm{mM} \mathrm{MgCl}_{2}$, and $75 \mathrm{~mm}$ sucrose was used. For storage and superfusion of slices, a physiological solution containing $125 \mathrm{~mm} \mathrm{NaCl}, 25 \mathrm{~mm}$ $\mathrm{NaHCO}_{3}, 2.5 \mathrm{~mm} \mathrm{KCl}, 1.25 \mathrm{~mm} \mathrm{NaH}_{2} \mathrm{PO}_{4}, 25 \mathrm{~mm}$ glucose, $2 \mathrm{~mm} \mathrm{CaCl}$, and $1 \mathrm{~mm} \mathrm{MgCl}$ was used. Slices were incubated at $34^{\circ} \mathrm{C}$ for $15-30 \mathrm{~min}$ after slicing and subsequently stored at room temperature. Both solutions were equilibrated with $95 \% \mathrm{O}_{2}-5 \% \mathrm{CO}_{2}$ gas mixture throughout the procedure.

\section{Electrophysiological recordings}

Whole-cell patch-clamp recordings were made with pipettes pulled from borosilicate glass capillaries ( $2 \mathrm{~mm}$ outer diameter and $1.5 \mathrm{~mm}$ inner diameter) with a resistance of 2.5-5 M $\Omega$. Pipettes were filled with a solution containing $120 \mathrm{~mm}$ potassium gluconate, $20 \mathrm{~mm} \mathrm{KCl}, 0.1 \mathrm{~mm}$ EGTA, $2 \mathrm{~mm}$ $\mathrm{MgCl}_{2}$, 4 mM Na 2 ATP, 0.5 mM GTP, 10 mM HEPES, 7 mm disodium phosphocreatine, and 0.1 or $0.2 \%$ biocytin, $\mathrm{pH} 7.2$ adjusted with $\mathrm{KOH}, \sim 300$ mOsm. $I-V$ experiments were performed with a pipette solution containing $110 \mathrm{~mm}$ cesium gluconate, $20 \mathrm{~mm} \mathrm{CsCl}, 10 \mathrm{~mm}$ EGTA, $2 \mathrm{~mm} \mathrm{MgCl}_{2}, 2 \mathrm{~mm}$ $\mathrm{Na}_{2} \mathrm{ATP}, 0.5 \mathrm{~mm}$ GTP, $1 \mathrm{~mm}$ QX-314 (lidocaine $N$-ethyl bromide), $10 \mathrm{~mm}$ HEPES, 8 mM TEA (tetraethylammonium)-Cl, and 0.1 or $0.2 \%$ biocytin, $\mathrm{pH}$ 7.2 adjusted with $\mathrm{CsOH}, \sim 300 \mathrm{mOsm}$. To determine the $I-V$ relationship for AMPA receptor (AMPAR)-mediated EPSCs $100 \mu \mathrm{M}$ spermine tetrahydrochloride was added to the pipette solution. Extracellular monopolar stimulation electrodes (resistance, $2-5 \mathrm{M} \Omega$ ) were filled with a HEPESbuffered $\mathrm{Na}^{+}$-rich solution containing $135 \mathrm{~mm} \mathrm{NaCl}, 5.4 \mathrm{~mm} \mathrm{KCl}, 1.8 \mathrm{~mm}$ $\mathrm{CaCl}_{2}, 1 \mathrm{~mm} \mathrm{MgCl}$, and $5 \mathrm{~mm}$ HEPES. NMDA receptor (NMDAR) and AMPAR-mediated currents were isolated in the presence of $20 \mu \mathrm{M}$ 6-cyano7-nitroquinoxaline-2,3-dione (CNQX) and 50-100 $\mu \mathrm{M}$ D-2-amino-5phosphonopentanoic acid (D-APV), respectively. In a subset of experiments, a washout of CNQX was required (see Fig. 6), and therefore only $10 \mu \mathrm{M}$ CNQX was bath-applied. The $\mathrm{GABA}_{\mathrm{A}}$ receptor antagonist $10 \mu \mathrm{M}$ bicuculline methiodide or 2-5 $\mu \mathrm{M}$ 4-[6-imino-3-(4methoxyphenyl)pyridazin-1-yl]butanoic acid hydrobromide (SR95531) was always added to the external solution except in experiments shown in Figure $2 D$ and supplemental Figure $2 C$ (available at www.jneurosci.org as supplemental material). All chemicals were purchased from Sigma-Aldrich, Tocris, and Merck.

Putative PIIs in acute slices were identified by the shape, size, and location of the soma at the granule cell layer ( $\mathrm{gcl}$ )-hilus border using infrared-differential interference contrast (IR-DIC) video microscopy (Koh et al., 1995) and their ability to discharge nonadapting trains of action potentials at high frequencies ( $\geq 120 \mathrm{~Hz} ; 600 \mathrm{pA}, 1 \mathrm{~s})$. The adaptation ratio was $>0.9$ determined from the ratio of the first and the last interspike interval during a train of action potentials (600 pA, $1 \mathrm{~s})$. In contrast, non-PIIs discharged trains of strongly adapting action potentials (adaptation ratio, $<0.5)$ at low frequencies $(\leq 60 \mathrm{~Hz} ; 600 \mathrm{pA}, 1 \mathrm{~s})$. All recordings were performed at $30-34^{\circ} \mathrm{C}$ except experiments during bath application of philanthotoxin (see below), which were made at room temperature $\left(20-22^{\circ} \mathrm{C}\right)$.

MFs were stimulated by placing an electrode in the hilus with a distance of $0-50 \mu \mathrm{m}$ to the gcl-hilus border near the soma of the recorded cell $(\geq 150$ $\mu \mathrm{m})$. MF inputs were reliably identified on the basis of their rapid time course and the characteristic frequency-dependent short-term dynamics (Geiger et al., 1997). Identity of the MF-PII synapses was further confirmed by their marked (2S,2R,3R)-2-(2,3-dicarboxycyclopropyl)glycine (DCGIV) sensitivity in a subset of experiments (23 PIIs). In all tested cells, the synaptic response evoked in the hilus was highly sensitive to bath-applied DCG-IV $(0.5 \mu \mathrm{M})$. Minimal stimulation intensities $(0.2 \mathrm{~ms} ;<10 \mathrm{~V})$ were applied to activate single MF inputs unless stated differently and defined as the voltage at which EPSCs were evoked with short latency with a probability of $<85 \%$. The PP was stimulated with a second electrode placed in the outer third of the molecular layer $(\mathrm{ml})$ (distance between both stimulation electrodes, $>200 \mu \mathrm{m}$ ). PP inputs were reliably identified on their slow time course (see Fig. $1 B$ ) and moderate DCG-IV sensitivity (22 PIIs) (supplemental Fig. $1 B$, available at www.jneurosci.org as supplemental material). To examine timing of action potentials generated by the PP and the MF inputs (see Fig. 1C), stimulation intensity was set to a value that generated action potentials with a probability of $\sim 50 \%(0.2 \mathrm{~ms}$; stimulus intensity, $<30 \mathrm{~V}$; applied at $0.2 \mathrm{~Hz}$ ). To evoke MF-mediated disynaptic EPSCs in PIIs on PP stimulation (supplemental Fig. 2, available at www.jneurosci.org as supplemental material), moderate intensities of three times the minimal stimulation strength were required.

Recordings were made using either Multiclamp 700B or Axopatch 200B amplifiers (Molecular Devices). The membrane potential was maintained at $-70 \mathrm{mV}$ in both voltage- and current-clamp experiments. Series resistance (Rs, 6-23 M $\Omega$ ) was compensated (voltage clamp, 7585\%; time lag, $10 \mu \mathrm{s}$; current clamp, 100\%; time lag, $1 \mu \mathrm{s}$ ). The Rs was regularly monitored and synaptic plasticity experiments were discarded if the Rs changed by $\pm 3 \mathrm{M} \Omega$. Signals were filtered at $10-20 \mathrm{kHz}$ (fourpole low-pass Bessel filter) and digitized at $20-40 \mathrm{kHz}$ using a CED 1401 digital interface (Cambridge Electronic Design). Pulse generation and data acquisition were performed using FPulse (U. Fröbe, Physiological Institute, University of Freiburg, Freiburg, Germany) running under Igor Pro 5.01 on a PC.

Pipettes (5-10 M $\Omega$ ) for perforated-patch recordings (current-clamp mode) were tip-filled with a gramicidin-free solution containing $100 \mathrm{mM}$ $\mathrm{KCl}, 5 \mathrm{~mm} \mathrm{MgCl}$, $10 \mathrm{~mm}$ HEPES, $5 \mathrm{~mm}$ glucose, $\mathrm{pH}$ adjusted to 7.2 with $\mathrm{KOH}$, and backfilled with the same solution containing gramicidin A (50 $\mu \mathrm{g} / \mathrm{ml}$; Merck) and Alexa Fluor 488 (50 $\mu \mathrm{M}$; Invitrogen). After establishing a cell-attached configuration, integrity of the perforated patch was monitored using epifluorescence illumination in $\sim 1-3$ min intervals throughout the experiment. EPSPs were only recorded after Rs had fallen below $100 \mathrm{M} \Omega$. Rs was fully compensated (see above), monitored every $\sim 2$ min intervals, and compensation was readjusted if necessary. Spontaneous breakthrough was identified by a sudden fluorescence labeling of the cell body (see Fig. 7A), accompanied by an abrupt reduction in Rs. Recording pipettes were removed after the experiment and cells were repatched with a pipette loaded with biocytin $(0.1 \%)$-containing solution for subsequent morphological identification (see below).

To test for synaptic plasticity in PIIs, two different burst frequency stimulation (BFS) protocols were used: First, for associative pairing (aBFS) (see Fig. 2), a BFS was applied to both pathways with the recorded PII held in the current-clamp mode. The BFS consisted of 12 bursts of stimuli delivered at a frequency of $0.3 \mathrm{~Hz}$; each burst was composed of 25 stimuli applied at a frequency of $30 \mathrm{~Hz}$ (Alle et al., 2001) consistent with the discharge frequency of GCs observed in vivo (Leutgeb et al., 2007). Stimulation of the PP preceded the stimulation of the MF pathway with a time interval $(\mathrm{d} t)$ of $10 \mathrm{~ms}$. Stimulus intensity was chosen to be subthreshold for both pathways (see above) at a membrane potential of -70 $\mathrm{mV}$ with minimal stimulation at MFs and close to threshold at the PP. The pairing protocol was repeated three times with a $30 \mathrm{~s}$ interval (Alle et al., 2001).

Second, a nonassociative BFS was applied to an individual pathway (see Figs. 3, 6, 7) and paired with a brief depolarizing suprathreshold current injection ( $0.2 \mathrm{~ms}$ duration) into the recorded PII held in the current-clamp mode $\left(V_{\text {hold }}=-70 \mathrm{mV}\right)$. The delay between the presyn- 
aptic stimulation and the postsynaptic depolarization was $0-2 \mathrm{~ms}$. The protocol was repeated three times with a $30 \mathrm{~s}$ interval (Alle et al., 2001). Posttetanic potentiation (PTP) was measured from peak amplitudes of evoked EPSCs $0-30 \mathrm{~s}$ after the BFS, and long-term potentiation (LTP) was determined from EPSCs 15-20 min after the BFS. LTP was usually monitored for 20-25 min. However, in four cells LTP recording was continued for up to $35 \mathrm{~min}$. In all experiments, LTP persisted until the end of the recording. EPSC amplitudes were normalized to the average EPSC obtained during the baseline period preceding the BFS and averaged over $30 \mathrm{~s}$ intervals. Data were not corrected for baseline noise.

\section{Data analysis}

For data analysis, Stimfit (http://www.stimfit.org/; courtesy of C. Schmidt-Hieber, University College London, London, UK; and P. Jonas, Physiological Institute, University of Freiburg, Freiburg, Germany) was used. Functional properties of evoked EPSCs were determined from averages of 30-50 traces. EPSCs were aligned to the beginning of the stimulus artifact. The rise time of evoked EPSCs was determined as the time interval between the points corresponding to 20 and $80 \%$ of the peak amplitude. The peak current was determined as the maximum within a window of 4-5 ms duration after the beginning of the stimulus artifact. The decay phase of the EPSCs was fitted with a monoexponential function $[\exp (-t / \tau)]$, using a nonlinear least-squares fit algorithm.

Spike probability (see Figs. $1 F, 2 F$ ) was determined as the ratio between the total number of applied pulses and the number of generated action potentials. Discharge frequency was quantified as the inverse of the mean interspike interval. Spike latency was determined as the time interval between the beginning of the stimulus artifact and the peak of the action potential.

To examine $I-V$ relationships, AMPAR- and NMDAR-mediated currents were measured at varying holding potentials $\left(V_{\text {hold }}=-80\right.$ to +80 $\mathrm{mV}$ ) chosen in a random sequence. AMPAR-mediated EPSCs at PP and MF synapses were recorded in the same cell. Peak amplitudes of average EPSCs (15-30 traces) were plotted against $V_{\text {hold }}$ and fitted with a linear or third-order polynomial function. Membrane potential values were not corrected for liquid junction potential. Extracellular stimulation artifacts in the figures were clipped for clarity. All data are shown as mean \pm SEM. Statistical significance was assessed using two-tailed paired or unpaired Student's $t$ test for repeated measures and independent samples, respectively. Alternatively, if data did not show normal distribution, a MannWhitney $U$ test or Wilcoxon's signed rank test was applied (statistic package from SigmaPlot, version 11.0). Significance levels are indicated as $p$ values.

\section{Post hoc histology}

Morphological analysis of interneurons was performed as reported previously (Bartos et al., 2001, 2002). All fast-spiking cells in this study were filled with biocytin during the recording, visualized, and identified morphologically as PIIs. A subset of the recorded cells was characterized neurochemically. Visualization of the cells involved one of the following two methods.

Peroxidase method. Slices were immersion-fixed in 2.5\% paraformaldehyde, $1.25 \%$ glutaraldehyde, and 15\% saturated picric acid in $100 \mathrm{~mm}$ phosphate buffer (PB), pH 7.4, and incubated in avidin-biotinylated peroxidase complex and 3,3'-diaminobenzidine tetrahydrochloride (DAB) as chromogen. The slices were embedded in Moviol and visualized neurons were examined on a bright-field light microscope.

Fluorescence method in combination with immunocytochemistry. Slices were fixed in $4 \%$ paraformaldehyde in $100 \mathrm{~mm}$ PB. After repeated rinses in PBS, pH 7.3, slices were first incubated in PBS containing 10\% normal goat serum (NGS) for $60 \mathrm{~min}$. Next, the primary monoclonal antibody against parvalbumin (rabbit-anti PV; Swant; 1:1000) was applied in PBS containing $0.3 \%$ Triton X-100 and $3 \%$ NGS for $24 \mathrm{~h}$ at $22^{\circ} \mathrm{C}$. After several rinses, slices were incubated in streptavidin conjugated with Alexa Fluor 647 or 488 (1:500; Invitrogen) and the secondary antibody conjugated with Cy3 (goat anti-rabbit; Jackson ImmunoResearch; 1:500) in PBS and $0.3 \%$ Triton X-100 for $24 \mathrm{~h}$ at $4^{\circ} \mathrm{C}$. Finally, slices were embedded in Moviol, and labeled neurons were examined with a confocal microscope (Zeiss, LSM 510). PIIs were identified on the basis of the predominant location of axon collaterals in the gcl. Using this approach, the two major PII types, basket and axo-axonic cells, cannot be reliably distinguished. However, because of their high abundance in the DG (Freund and Buzsáki, 1996), our sample of PIIs will mostly comprise basket cells and may include some axo-axonic cells.

\section{Results}

\section{PP- and MF-mediated excitation onto PIIs have different kinetic properties}

We obtained whole-cell patch-clamp recordings of EPSCs in PIIs evoked by extracellular stimulation of two convergent inputs, the PP and the MF pathway in rat hippocampal slice preparations (Fig. 1). PIIs were identified during recordings on the basis of their nonadapting, high-frequency trains of action potentials generated in response to depolarizing current injection (Fig. 1A). Cells were filled with biocytin during the recordings and identified as PIIs if the axon was predominantly located in the gcl (Figs. $1 A, D, 2 C$; supplemental Fig. $6 C$, available at www.jneurosci.org as supplemental material). In a subset of the recorded PIIs, we tested for parvalbumin expression and found that all seven of the seven recorded cells were immunopositive (Fig. $1 D$; supplemental Fig. 6C, available at www.jneurosci.org as supplemental material).

For stimulation of the pathways, one electrode was positioned in the outer third of the $\mathrm{ml}$ and another one at the gcl-hilus border (Fig. 1A). The two pathways were stimulated separately in an alternating pattern to monitor individual PP- and MF-EPSCs (see Materials and Methods). To confirm the identity of the stimulated fibers, we tested the effect of metabotropic glutamate receptor (mGluR) agonists known to differentially modulate transmission at these pathways (supplemental Fig. 1, available at www.jneurosci.org as supplemental material). Bath application of the group III mGluR agonist L-(+)-2-amino-5-phosphonopentanoic acid (L-AP4) inhibited ml-evoked EPSCs (amplitude reduction, $67.3 \pm 5.6 \%$; seven cells; $p<0.001$ ) but had only weak effects on EPSCs elicited at the gcl-hilus border (13.8 $\pm 5.6 \%$; four cells) (supplemental Fig. $1 A$, available at www.jneurosci.org as supplemental material). In contrast, the group II mGluR agonist DCG-IV strongly reduced EPSCs elicited at the gcl-hilus border $(61.8 \pm 3.4 \%$; seven cells; $p=0.011)$ (supplemental Fig. $1 B$, available at www.jneurosci.org as supplemental material) but had a much weaker influence on those elicited in the $\mathrm{ml}(26.7 \pm 10.2 \%$; nine cells; $p=0.377)$. Thus, stimulation in the outer $\mathrm{ml}$ activated predominantly lateral PP fibers, whereas gclhilus border stimulation recruited MF axons (Macek et al., 1996; Dietrich et al., 1997).

Electrotonic filtering contributes markedly to attenuation of synaptic signals (Rall and Segev, 1985; Major et al., 1993; Häusser and Roth, 1997; Bartos et al., 2001; Nörenberg et al., 2010). Therefore, PP-mediated ESPCs originating at more distal dendritic locations are expected to show stronger attenuation at the soma than those mediated by proximal MF inputs. Indeed, EPSCs elicited by stimulation of the two pathways could be reliably distinguished on the basis of their kinetic properties (Fig. $1 B)$. EPSCs evoked at MF-PII inputs were rapid (Geiger et al., 1997), whereas PP-mediated EPSCs showed a much slower time course. The rise time (RT) was significantly longer (PP, $0.94 \pm$ $0.09 \mathrm{~ms}$, vs MF, $0.55 \pm 0.04 \mathrm{~ms} ; 15$ cells; $p=0.001)$, and the decay time constant $\left(\tau_{\text {decay }}\right)$ was two times higher for PP- than for MFEPSCs $(5.9 \pm 0.4$ vs $2.3 \pm 0.3 \mathrm{~ms} ; p<0.001)$ (Fig. $1 B$ ). However, the large difference in the decay indicates that additional factors, such as differential expression of postsynaptic AMPAR subtypes, may also play a role (see below). 
A

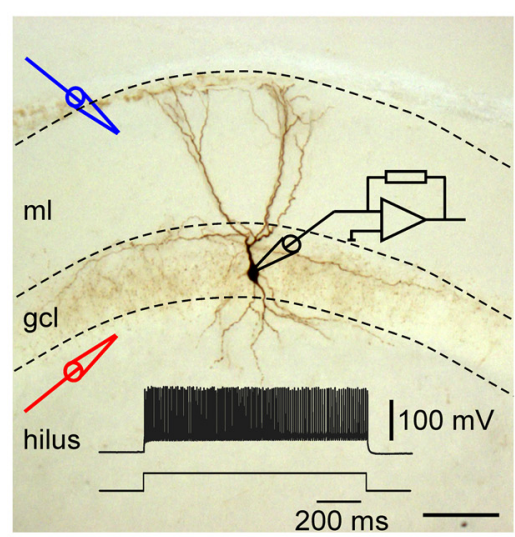

D

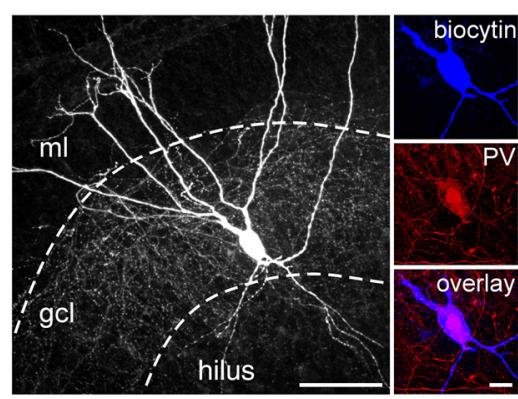

B

PP
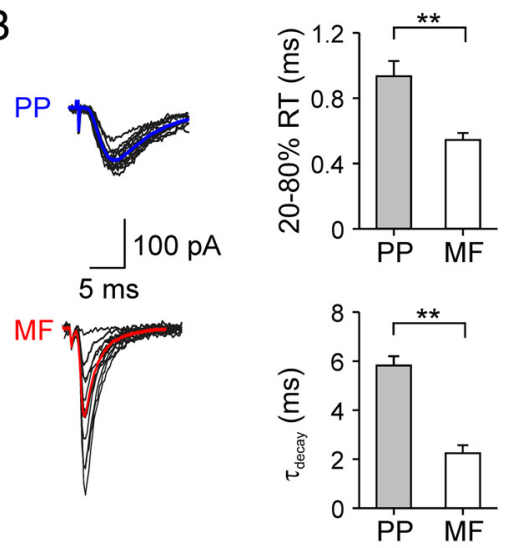

$\mathrm{E}$

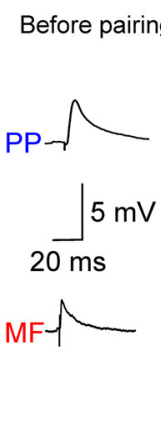

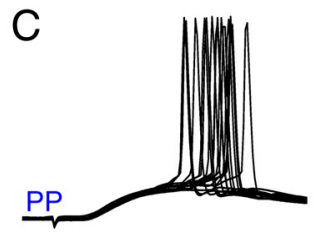

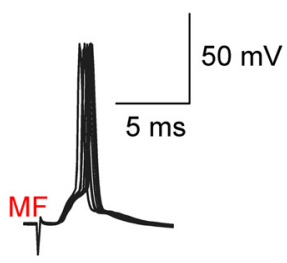

$\mathrm{F}$
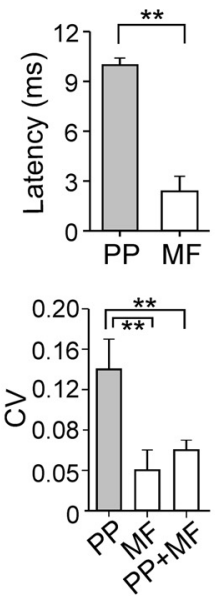

Figure 1. Convergence of slow perforant path- and fast mossy fiber-mediated excitation leads to reliable and precise activation of DG perisomatic inhibitory interneurons. $\boldsymbol{A}$, Illustration of the EPSC recording configuration. Black electrode, Somatic whole-cell recording from a PII; blue electrode, placed in the outer ml for extracellular stimulation of PP fibers; red electrode, positioned at the $\mathrm{gcl}$-hilus border to stimulate MFs. Cell was filled with biocytin during the recording and visualized with DAB as chromogen. Dense axonal arbor in the gcl identify this cell as PII. Inset, Fast-spiking phenotype of the same PII (600 pA). The dashed lines indicate the borders of the gcl and the ml. B, Superimposed individual EPSCs (black) in response to PP (top) or MF (bottom) stimulation, recorded in the same PII. Note fluctuation in the peak amplitude of MF-mediated EPSCs. Stimulation intensity was set to 1.5 times the minimal stimulation. The blue and red traces are averaged EPSCs. Right, Bar charts provide comparison of 20-80\% RT and decay time constant ( $\left.\tau_{\text {decay }}\right)$ of average EPSCs. C, Left, Superimposed action potentials during PP (top) or MF (bottom) stimulation recorded in current-clamp mode. Right, Comparison of mean values and the CV of evoked action potential latencies. D, Left, Projection of a confocal image stack of another recorded PII. The biocytin-filled interneuron was visualized using streptavidin-conjugated Alexa 647 dye. Right, High-power confocal images show immunoreactivity for parvalbumin (PV) in the soma of the same cell. $\boldsymbol{E}$, Pairing of $\mathrm{PP}$ - and MF-EPSPs with varying relative latencies ( $\mathrm{d} t=t_{\mathrm{MF}}-t_{\mathrm{PP}}$ ). PP stimulation was applied at $t=0 \mathrm{~ms}$ and timing of the MF stimulation (asterisk) was changed in $5 \mathrm{~ms}$ steps between -10 and $+20 \mathrm{~ms}$. The left panel shows the subthreshold PP- and MF-EPSPs. On the right, superposed responses illustrate the efficient initiation of action potentials by the paired stimuli with a $10 \mathrm{~ms}$ relative delay. $\boldsymbol{F}$, Bar chart summary of action potential probability as a function of the delay $\mathrm{d} t$ between PP and MF stimulation ( 5 cells). Data were normalized to values obtained at $\mathrm{d} t=10 \mathrm{~ms}$ and fitted with a Gaussian function (dashed line). Scale bars: $\boldsymbol{A}, \boldsymbol{D}$, left, $100 \mu \mathrm{m} ; \boldsymbol{D}$, right, $20 \mu \mathrm{m}$. ${ }^{* *} p<0.01$, two-tailed Student's $t$ test. The bars represent means \pm SEM.

The distinct EPSC kinetics suggested that synaptic activation of PIIs by the two inputs could result in differences in spike timing. We tested this hypothesis by recording PII discharges in response to extracellular stimulation (see Materials and Methods). MF activation with extracellular stimulation intensities that resulted in $\sim 50 \%$ discharge probability, resulted in the generation of action potentials with short latencies and high temporal precision (Fig. 1C). The mean latency was $2.4 \pm 0.9 \mathrm{~ms}$, and the precision, quantified by the coefficient of variation $(\mathrm{CV})$ of spike latencies, was $0.04 \pm 0.02$ (five cells). In contrast, PP stimulation elicited action potentials with significantly longer latencies and lower temporal precision (latency, $10.0 \pm 0.5 \mathrm{~ms} ; 17$ cells; $p<$ $0.001 ; C V, 0.14 \pm 0.03 ; p<0.001$ ) (Fig. $1 C$ ). Thus, differences in the kinetics of PP- and MF-mediated EPSCs translate into different timing and precision of action potential initiation in PIIs.

\section{Convergence of PP- and MF-mediated excitation leads to} reliable and precise activation of PIIs

The activation of PIIs by PP stimulation seems to be incompatible with the assumed reliable and precise recruitment of synaptic inhibition in the DG circuitry (Bartos et al., 2001, 2002, 2007; Jonas et al., 2004). However, in intact networks, afferent activation can recruit principal cells and produce excitation in PIIs via their recurrent collaterals, the MFs (Acsády et al., 1998). Therefore, to analyze how the two excitatory inputs may converge to activate PIIs, we stimulated the PP and MFs together within a narrow time window (Fig. $1 E, F$ ). The stimulus intensity at individual pathways was chosen to be subthreshold, with close to threshold at the PP and minimal stimulation at MFs (see Materials and Methods). The mean peak amplitude of PP-EPSCs was $287.7 \pm 96.1 \mathrm{pA}$ (six cells). MF-EPSCs had amplitudes of $134.6 \pm$ $23.3 \mathrm{pA}$ (six cells) comparable with that of unitary EPSCs (Alle et al., 2001).

We assessed integration of the two inputs by systematically changing the delay $(\mathrm{d} t)$ between the MF and the PP stimulation from -10 to $25 \mathrm{~ms}$ at $5 \mathrm{~ms}$ steps (Fig. $1 \mathrm{E}$ ). With delay values between 0 and $15 \mathrm{~ms}$, EPSPs from the two inputs showed substantial summation. Under this condition, action potentials were generated with short latency and high precision after MF stimulation (PP stimulation alone: $\mathrm{CV}, 0.14 \pm 0.03$; vs $\mathrm{MF}$ and $\mathrm{PP}$ stimulation: $\mathrm{CV}, 0.06 \pm 0.01 ; p<0.01 ; 17$ and 10 cells, respectively). Maximal discharge probability $(\sim 50 \%)$ was achieved at a MF input delay of $10 \mathrm{~ms}$ but dropped rapidly with shorter and longer delays (Fig. 1E,F). Thus, recurrent excitation, even by a single GC, enhances the temporally precise recruitment of PIIs by the entorhinal input. 
A
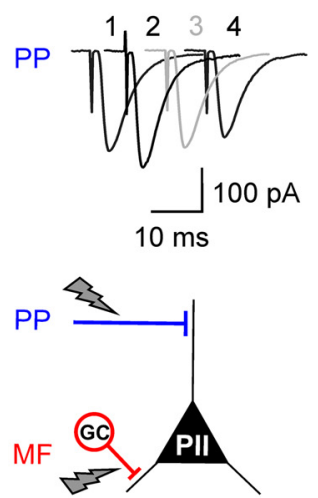

1234

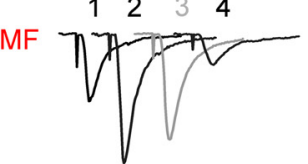

C

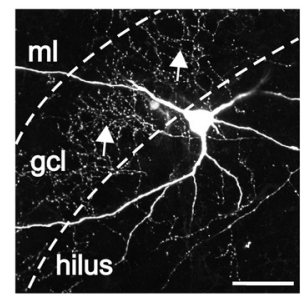

B
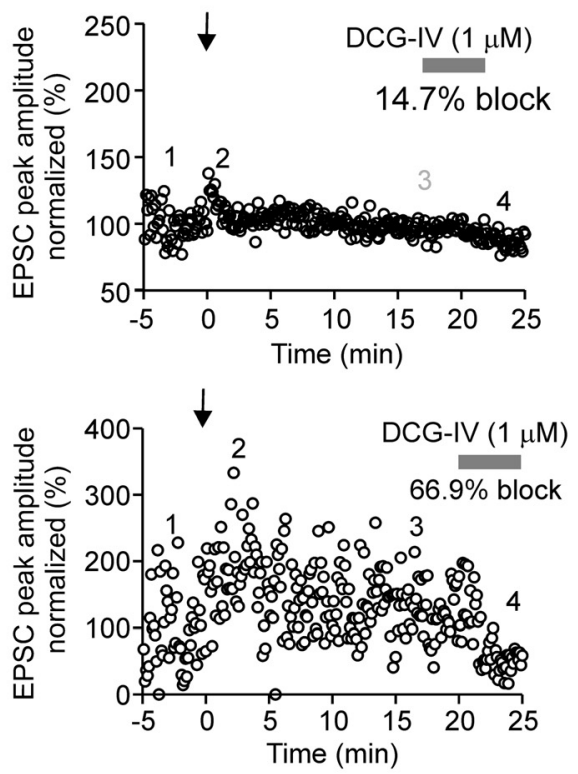

D

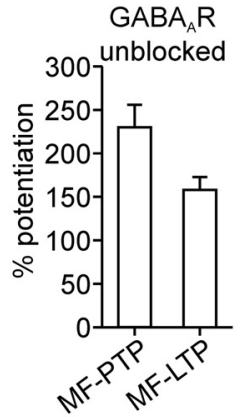

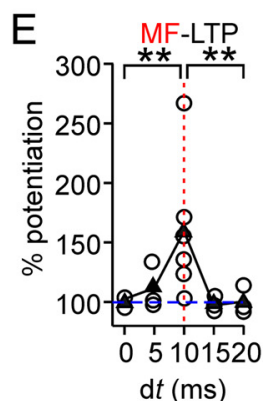
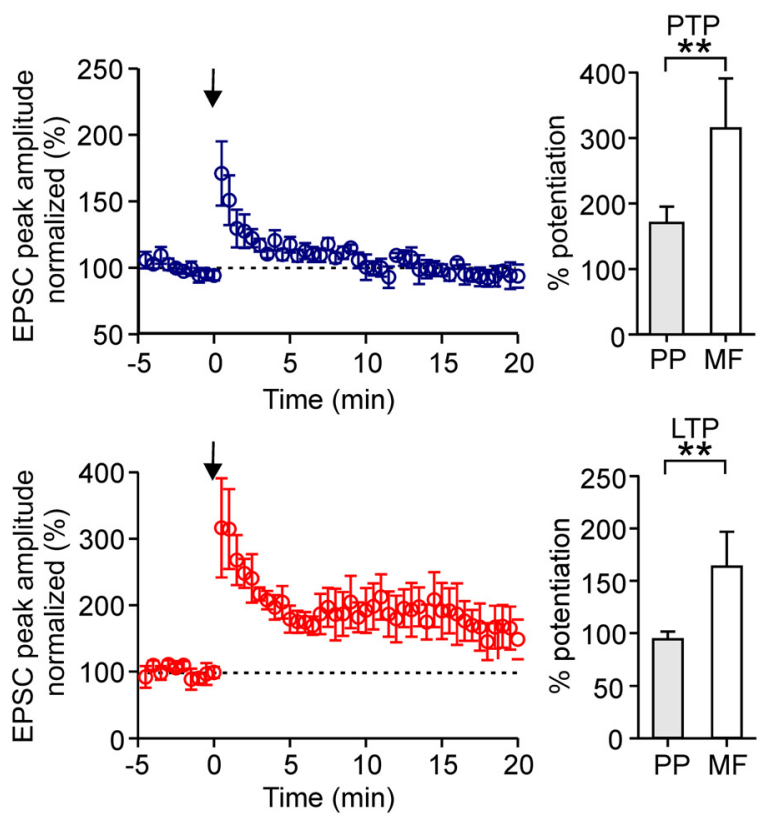

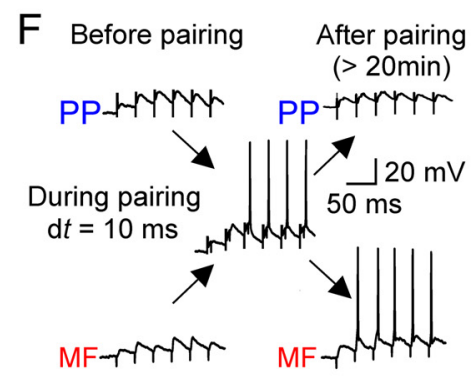

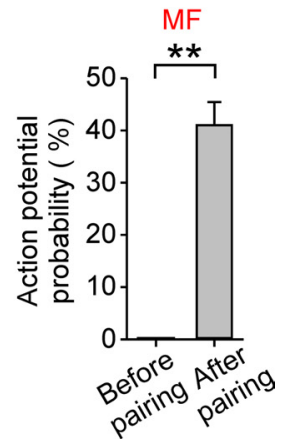

Figure 2. Associative pairing of perforant path and mossy fiber inputs in perisomatic inhibitory interneurons induces Hebbian LTP selectively at mossy fiber synapses. $A$, To test plasticity, an aBFS pattern was applied, in which PP stimulation preceded MF stimulation by $10 \mathrm{~ms}$ (spike probability during pairing in this experiment $71 \%$, corresponding to a mean discharge frequency of $24 \mathrm{~Hz}$ ). The panel shows average EPSCs in response to PP (top traces) or MF (bottom traces) stimulation before the aBFS (1), immediately after the aBFS, during PTP (2), 15-20 min after the aBFS induction (gray) (3), and after application of $1 \mu \mathrm{m} \mathrm{DCG-IV} \mathrm{(4).} \mathrm{B,} \mathrm{Left,} \mathrm{Normalized} \mathrm{EPSC} \mathrm{peak} \mathrm{amplitudes} \mathrm{are} \mathrm{plotted} \mathrm{against} \mathrm{time} \mathrm{from} \mathrm{a} \mathrm{single} \mathrm{experiment.} \mathrm{PP-} \mathrm{(top} \mathrm{plot)} \mathrm{and} \mathrm{MF-mediated} \mathrm{EPSCS} \mathrm{(bottom} \mathrm{plot)} \mathrm{were}$ recorded in an alternating manner in the PII ( $800 \mathrm{~ms}$ apart). EPSCs were normalized to the baseline. The arrows indicate the time of the pairing protocol. Middle, Summary plots of the experiments from five cells. Each circle represents the average of EPSCs over 30 s intervals for five cells; PP-EPSC amplitudes are in blue, and MF-EPSCs are in red. Right, Bar graphs compare the extent of PTP and LTP at the two pathways. C, Morphology of the PII from which data are shown in $\boldsymbol{A}$ and $\boldsymbol{B}$. The arrows point to axon collaterals in the gcl. The dashed lines indicate borders of the gcl toward the $\mathrm{ml}$ and the hilus. D, Summary bar graph illustrates the level of PTP and LTP at MF-PIl synapses induced by associative pairing in the presence of GABAergic transmission (3 cells). E, Summary plot shows the potentiation of the EPS ( peak amplitude, measured 15-20 min after aBFS application, as a function of the relative latency between the PP and MF stimulation (dt). The open circles represent data from individual experiments, and the filled triangles represent the mean values for every $\mathrm{dt}$.F, MF-EPSPs but not PP-EPSPs can recruit Plls after LTP induction. Brief trains ( $30 \mathrm{~Hz}$ ) of EPSPs evoked by PP (top traces) or MF (bottom traces) stimulation before pairing (left) and $>20$ min after LTP induction (right). Responses to the first 6 of 25 consecutive pulses are shown. Right, Summary bar graph of the probability of action potential generation in response to burst stimulation of MF synapses before and after the pairing from five cells. Scale bar, $100 \mu \mathrm{m} .{ }^{* *} p<0.01$, two-tailed Student's $t$ test and Mann-Whitney $U$ test. Average measurements are given as mean \pm SEM.

Does the relative delay of $10 \mathrm{~ms}$ for efficient summation of the two inputs correspond to the disynaptic latency along the PPGC-PII pathway? To address this question, we tested the recruitment of recurrent excitation of PIIs by PP activation alone (supplemental Fig. 2, available at www.jneurosci.org as supplemental material). A moderate increase in the stimulus intensity of the PP (see Materials and Methods) elicited a response composed of a monosynaptic EPSC with a mean latency of $1.8 \pm 0.1 \mathrm{~ms}$ followed by a disynaptic EPSC with a markedly longer latency of $9.5 \pm 0.4 \mathrm{~ms}$ (nine cells; $p=0.003$ ) (supplemental Fig. $2 A, B$, available at www.jneurosci.org as supplemental material). Although bath application of DCG-IV produced only a moderate reduction in the monosynaptic EPSC (amplitude reduction, $16.1 \pm 5.9 \%)$, it substantially blocked the disynaptic EPSC (64.4 $\pm 7.1 \%$; 10 cells) (supplemental Fig. 2 B, available at www. jneurosci.org as supplemental material), confirming its MFmediated nature. Thus, the relative delay of $10 \mathrm{~ms}$ corresponds well to the disynaptic latency and suggests that integration of convergent inputs in PIIs is optimized for network structure.

Pairing of the two afferent inputs in one PII induces plasticity at MF synapses

Can associative activation of the two afferent inputs lead to longlasting changes in the efficacy of MF synapses? To address this question, we repeatedly paired subthreshold PP and MF inputs in PIIs with the optimal $10 \mathrm{~ms}$ interval by applying an aBFS (Alle et al., 2001) (Fig. 2). During delivery of the aBFS pattern, action potentials were generated on most but not all stimuli because of variability in the amplitude of EPSPs. The mean discharge frequency of the PIIs was $18.2 \pm 5.5 \mathrm{~Hz}$, reflecting a discharge prob- 

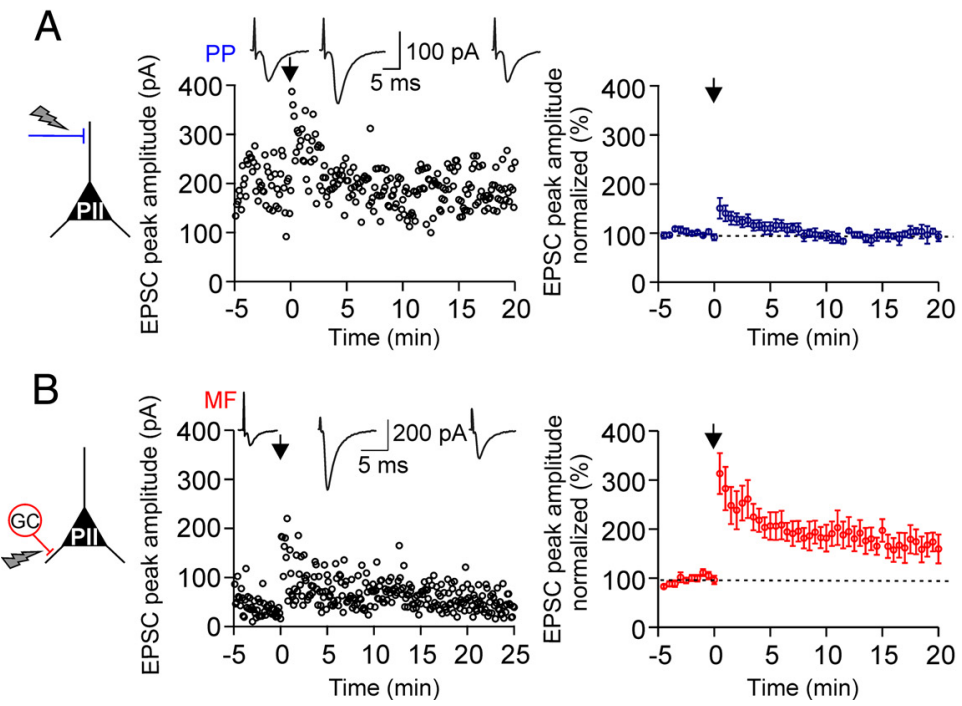

Figure 3. Afferent-specific expression of LTP in perisomatic inhibitory interneurons. A, A nonassociative BFS applied to PP-PII synapses in combination with brief suprathreshold depolarizations in the postsynaptic cell (see Materials and Methods) induces PTP but not LTP. Left, Individual EPS ( peak amplitudes from a single experiment are plotted against time before and after pairing. The nonassociative BFS was applied at $t=0 \mathrm{~ms}$ (arrow). Insets on top, Average EPSCs (30 traces) during the baseline period (left), during PTP (middle), and 15-20 min after the induction protocol (right). Right, Summary time course of EPSC peak amplitudes evoked at PP-PII synapses (blue) before and after pairing from eight cells. EPSCs were averaged over 30 s intervals and normalized to baseline values. $\boldsymbol{B}$, Corresponding data for MF-PII synapses. At the MF input, pairing resulted in a PTP followed by a marked LTP (10 cells). C, D, Summary bar graphs comparing the effect of the applied nonassociative BFS on the peak amplitude of average EPSCS during the PTP $(\boldsymbol{C})$ and the LTP phase $(\boldsymbol{D})$ evoked by extracellular stimulation of the PP or the MF inputs. ${ }^{* *} p<0.01$; two-tailed Student's $t$ test and Mann-Whitney $U$ test. Average measurements are represented as mean \pm SEM.

ability of $61 \%$. This activity is consistent with firing properties of hippocampal basket cells during network activity observed in vivo (Bragin et al., 1995; Tukker et al., 2007).

Application of the aBFS resulted in a marked PTP of synaptic responses at MF synapses (316.3 $\pm 74.6 \%$ of baseline amplitude, measured for $30 \mathrm{~s}$ after pairing; $p=0.006$ ) followed by a LTP (164.4 $\pm 32.3 \%, 15-20$ min after pairing; for five of six cells; $p=$ 0.001 ) (Fig. 2 B). Potentiated EPSCs could be blocked by DCG-IV 20 min after LTP induction, confirming that they were mediated by MFs (three of three cells) (Fig. 2A,B). In contrast, PP-PII synapses showed only PTP, which was significantly smaller than at MF inputs $(170.9 \pm 24.2 \% ; p=0.006)$. The peak amplitude of potentiated EPSCs declined back to control values within $\sim 10$ min after pairing and no LTP was induced (PP-EPSC amplitude, 95.1 $\pm 6.3 \%$; 15-20 min after pairing; six cells) (Fig. 2B).

Changing the delay between PP and MF from the optimal $10 \mathrm{~ms}$ to values $\leq 5$ or $\geq 15 \mathrm{~ms}$ resulted in a significant reduction in the probability of action potential generation to $<30 \%$ for 5 and $15 \mathrm{~ms}$ delays and $<3 \%$ for 0 and $20 \mathrm{~ms}$ delays. The reduced discharge probability during pairing was paralleled by a diminished LTP at MF-PII synapses $(103.0 \pm 3.1 \%$; 11 cells; $p<0.001)$ (Fig. $2 E$ ), indicating that LTP induction is dependent on action potential generation. To further test this, we hyperpolarized postsynaptic PIIs in subsequent experiments to $-90 \mathrm{mV}$, to prevent action potential generation during the induction paradigm with the optimal $10 \mathrm{~ms}$ delay. This manipulation also prevented LTP induction (100.4 \pm 1.7\%; three cells) (supplemental Fig. 3, available at www.jneurosci. org as supplemental material), confirming that associative LTP re quires the generation of action potentials in DG PIIs (Alle et al., 2001).

To examine how synaptic plasticity influences the recruitment of PIIs, we applied short bursts of 25 pulses at $30 \mathrm{~Hz}$ to MFs at minimal stimulus intensities (Fig. $2 F$ ). During the baseline period,
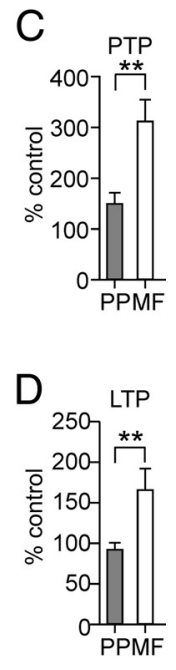

the stimulus train resulted in a facilitating sequence of EPSPs, but no action potentials were generated. However, after LTP was induced, PIIs readily generated action potentials in response to minimal stimulation of the potentiated MF input (40.0 \pm $4.4 \%$ spike probability during a burst; four cells; measured $>20$ min after pairing). No similar effect was observed at the PP input (Fig. $2 F$ ). Thus, associative LTP enables the efficient recruitment of PIIs even by a single convergent MF input.

All the above experiments were performed in the presence of $\mathrm{GABA}_{\mathrm{A}}$ receptor antagonist SR95531 to isolate excitatory synaptic responses. However, inhibition is known to control the induction of synaptic plasticity (Paulsen and Moser, 1998). It is therefore an important question whether associative plasticity can develop under conditions of intact $\mathrm{GABA}_{\mathrm{A}}$ receptor-mediated synaptic inhibition. Therefore, we repeated the experiments using the associative pairing paradigm of the two inputs with the optimal delay in the absence of SR95531. Under this condition, we observed a marked LTP at MF-PII synapses (150.3 $\pm 15.2 \%$; three cells) (Fig. 2D) similar to the situation with inhibition blocked $(164.4 \pm 32.3 \%)$

(Fig. $2 B$ ), demonstrating that induction of MF-PII LTP can take place under physiological conditions when inhibition is intact (see also supplemental Fig. 2C, available at www.jneurosci.org as supplemental material).

We have shown above that stimulation of the PP at increased intensities can recruit GCs, produce disynaptic MF-EPSCs and precisely timed action potentials in PIIs (Fig. 1 E, F; supplemental Fig. 2, available at www.jneurosci.org as supplemental material). Can this stimulation protocol also lead to long-lasting changes in synaptic efficacy at MF-PII synapses? To address this question, we stimulated the PP with moderate intensities (see Materials and Methods). As expected, this stimulus protocol evoked compound EPSCs composed of a monosynaptic PP-EPSC followed by disynaptic DCG-IV-sensitive MF-EPSC (supplemental Fig. 2C, available at www.jneurosci.org as supplemental material). When BFS was applied at these intensities to the PP input alone, it induced LTP of the disynaptic MF-mediated EPSC, but not the monosynaptic PP-mediated component (disynaptic EPSC, $239.8 \pm 26.3 \%$; monosynaptic, $88.8 \pm 2.5 \%$; five cells; $p=0.008$ ), further supporting our hypothesis that conditions for synaptic plasticity at MF-PII synapses is optimized for network structure.

In summary, these experiments identified a synapse-specific, associative plasticity in PIIs induced by pairing of convergent PP and MF excitatory inputs.

\section{Afferent-specific expression of synaptic plasticity in PIIs}

The presence of plasticity at MF and its apparent absence at PP inputs was an intriguing finding. Therefore, we examined whether a nonassociative Hebbian stimulus protocol can induce LTP at the two pathways. We applied a BFS selectively to either the naive MF or to the naive PP input in combination with brief suprathreshold depolarizing pulses in the postsynaptic PII (Fig. 3). 
A

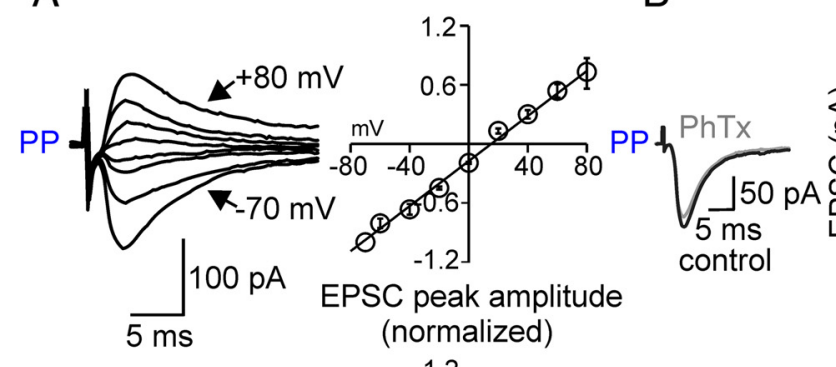

B

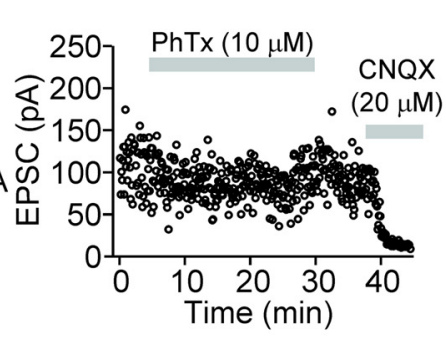

C

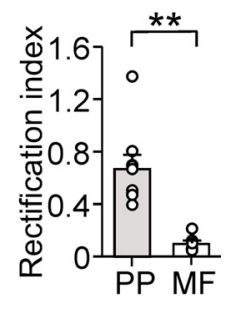

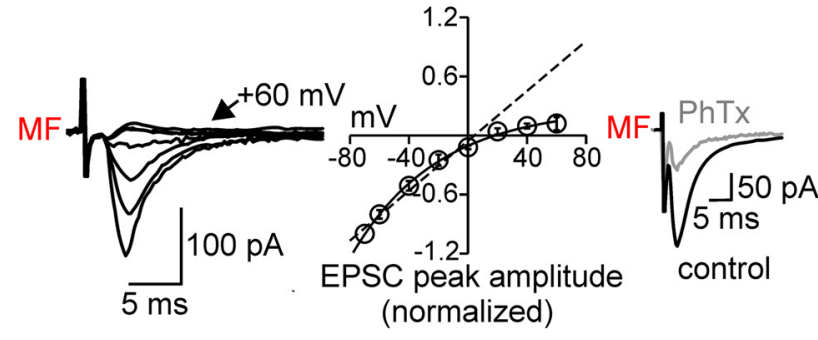
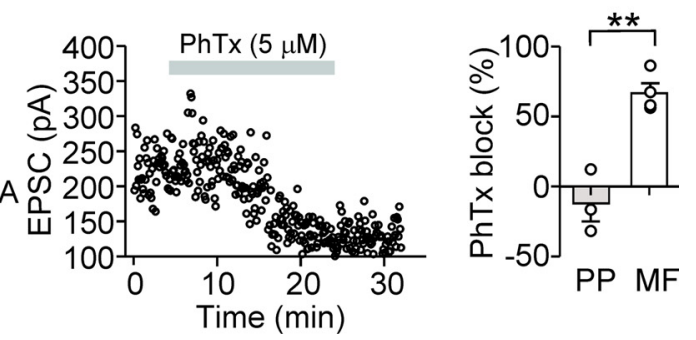

Figure 4. Afferent-specific expression of $\mathrm{Ca}^{2+}$-impermeable and $\mathrm{Ca}^{2+}$-permeable AMPA receptors in perisomatic inhibitory interneurons. A, Left, Average EPSCs from 30 single traces evoked at PP-PII (top) and MF-PII (bottom) inputs for various holding potentials ( 10 or $20 \mathrm{mV}$ increments). Right, Current-voltage ( $/$-V relationship for PP- (top; 9 cells) and MF-mediated (bottom; 6 cells) EPSCs in PIls. Peak amplitudes of the average EPSCs were plotted against the holding potential. The black curves represent the linear (top) or polynomial function (bottom) fitted to the data. The inwardly rectifying /-V relationship at MF-PII synapses is a hallmark of CP-AMPARs (Geiger et al., 1995; Tóth and McBain, 1998). B, MF-mediated EPSCs are blocked by 5-10 $\mu$ m PhTX-433, a selective CP-AMPAR blocker (Tóth and McBain, 1998). Left, Superposed average EPSCs evoked at PP-PII (top) and MF-PII (bottom) synapses under control conditions (black traces) and during bath application of PhTx-433 (gray traces). Right, Time course of the PhTx-433 effect; peak amplitude of EPSCs evoked at the PP (top) and the MF inputs (bottom) are plotted against time. The gray bars represent the wash-in of PhTX-433 and, at a later phase, CNQX. C, Top bar graph, Summary of the rectification index (EPSC at $+60 \mathrm{mV} / \mathrm{EPSC}$ at $-60 \mathrm{mV})$ for EPSCs evoked at PP-PII (9 cells) and MF-PII synapses ( 6 cells). Bottom bar graph, Comparison of the average block of PhTx-433 on PP- (3 cells) and MF-mediated EPSCs ( 4 cells). ${ }^{* *} p<0.005$; Mann-Whitney U test. Average measurements represent mean \pm SEM.

When the BFS was delivered to MF inputs, a PTP developed $(313.0 \pm 41.6 \% ; 10$ cells; $p<0.001)$ (Fig. $3 B, C)$, followed by a marked LTP in all tested cells $(166.4 \pm 25.7 \% ; 10$ cells; $p<0.001)$ (Fig. $3 B, D$ ). EPSCs could be blocked by DCG-IV confirming their MF-mediated nature (three of three cells). The amplitude of EPSCs evoked by control stimuli applied to the PP remained constant throughout the experiment indicating that the induction and expression of plastic changes at MF-PII synapses did not influence transmission at PP-PII synapses. In contrast, when the BFS was delivered to the PP, only a shortlasting PTP was observed $(150.6 \pm 21.0 \%$; eight cells; $p=$ 0.004 ) (Fig. $3 A, C$ ) but no LTP developed in any of the recorded cells $(97.7 \pm 1.7 \% ; p>0.95)$ (Fig. $3 A, D)$. Control stimuli applied to MFs elicited EPSCs with constant amplitude, demonstrating that stimulation of the PP input alone is not sufficient for LTP induction at the convergent MF input. These results further indicate the synapse-specific expression of plasticity in DG PIIs.

The fact that LTP was not induced at PP inputs may result from the general absence of plasticity in the examined cells. Therefore, in three additional cells, we applied a second BFS to the MFs after the first BFS had been delivered to the PP. In all cells tested, LTP was induced at MF inputs demonstrating their ability to express synaptic plasticity (PTP, $353.5 \pm 69.9 \%$; LTP, $201.1 \pm$ $70.2 \%$ ) (supplemental Fig. $4 A$, available at www.jneurosci.org as supplemental material). Conversely, in three other PIIs, a second BFS was applied to the PP after LTP has been induced and monitored for $20 \mathrm{~min}$ at MF inputs. Consistent with the above results, no LTP was induced at PP-PII synapses by the second BFS applied to the PP in any of these cells (PTP, $164.9 \pm 24.5 \%$; LTP, $100.5 \pm 5.2 \%$ ) (supplemental Fig. $4 B$, available at www. jneurosci.org as supplemental material). These results demonstrate that a nonassociative BFS (i.e., stimulation of one pathway alone), similar to associative pairing of PP and MF inputs (Fig. $2 B$ ), leads to the induction of input-specific LTP at MF-PII synapses but not at PP-PII synapses.

The large electrotonic distance in fast-spiking PIIs (Fig. $1 A$ ) could produce attenuation of backpropagating action potentials. This may, in turn, reduce synaptic plasticity at distal synaptic inputs as previously shown for cortical pyramidal cells (Sjöström and Häusser, 2006). In this case, stronger depolarization of dendrites could unmask plasticity at PP synapses. We tested this possibility by increasing the BFS frequency from 30 to $50 \mathrm{~Hz}$. This manipulation is expected to enhance summation of EPSPs and to increase depolarization of distal dendrites (Sjöström and Häusser, 2006). However, no long-lasting changes in EPSC amplitude were observed at PP inputs after this induction protocol $(91.0 \pm 3.0 \%$; three cells; $15-20$ min after BFS application). We also tested if cooperativity of synaptic inputs can increase dendritic depolarization and lead to LTP (supplemental Fig. 5, available at www.jneurosci.org as supplemental material). Two independent PP inputs were activated simultaneously by applying a strong and a weak stimulus (Nicoll et al., 1988), but LTP was not induced at either of the stimulated inputs (supplemental Fig. 5C, available at www.jneurosci.org as supplemental material). These convergent results suggest that PP-PII synapses do not express LTP under our conditions. However, additional investigations are needed to examine whether other stimulus paradigms (e.g., different burst patterns) or different modulatory states may result in plasticity at PP-PII synapses.

\section{Afferent-specific expression of $\mathrm{Ca}^{2+}$-permeable AMPAR and NMDAR in PIIs}

To investigate the mechanisms that contribute to the afferentspecific plasticity at PII inputs, we asked whether the presence or 
A
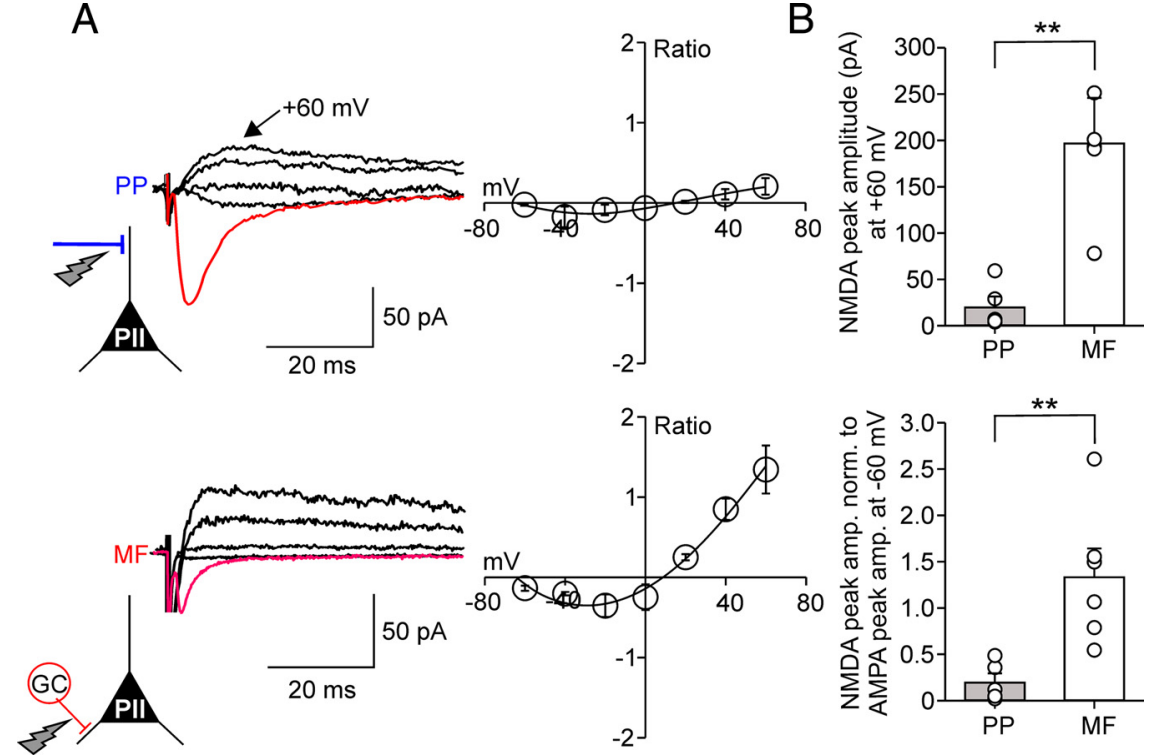

Figure 5. The ratio between NMDA receptor- and AMPA receptor-mediated components is larger at mossy fiber than at perforant path input synapses onto perisomatic inhibitory interneurons. $\boldsymbol{A}$, Left in red, AMPAR-mediated average EPSCs ( 30 single traces) at a $V_{\text {hold }}$ of $-60 \mathrm{mV}$ at PP-PII (top) and MF-PII (bottom) synapses (in the absence of NMDAR blocker). Superimposed in black, Average NMDAR-mediated EPSCs evoked at four selected holding potentials $(-20,+20,+40$, and $+60 \mathrm{mV})$ after bath application of the AMPAR blocker CNQX $(20 \mu \mathrm{m})$. Right, I-V relationship for the PP-PII (top; 5 cells) and the MF-PII synapsemediated (bottom; 6 cells) NMDA component normalized to the AMPAR-mediated component at $-60 \mathrm{mV}$. The black curves represent third-order polynomial functions fitted to the data. $\boldsymbol{B}$, The top bar graph summarizes the peak amplitude data for NMDAR-mediated EPSCs recorded at $+60 \mathrm{mV}$ evoked at PP-PII and MF-PII synapses. The bottom bar graph compares the NMDAR-mediated component at $+60 \mathrm{mV}$ normalized to EPSCs evoked by AMPAR activation at $-60 \mathrm{mV}$ for the two pathways. ${ }^{* *} p<0.01$, two-tailed Student's $t$ test. Average measurements represent mean \pm SEM.
AMPAR-mediated components. MF synapses elicited large average NMDARmediated currents at $+60 \mathrm{mV}(197.8 \pm 48.1$ pA; six cells) (Fig. 5A), whereas corresponding NMDA currents elicited at PP synapses had a substantially smaller peak amplitude $(20.8 \pm 10.7$ pA; five cells; $p=0.004)$ (Fig. $5 B)$. This difference may be caused by strong electrotonic filtering of distally evoked PP signals. We therefore determined the ratio between NMDARmediated currents at a $V_{\text {hold }}$ of $+60 \mathrm{mV}$ and AMPAR currents at $-60 \mathrm{mV}$. Consistent with the difference in the peak amplitudes, the NMDA/ AMPA ratio was sevenfold lower for PP than for MF synapses (PP, $0.21 \pm 0.1$, five cells; vs $\mathrm{MF}$, $1.3 \pm 0.3$, six cells; $p=0.009$ ) (Fig. $5 B$ ), indicating that NMDAR-mediated contribution to PII excitation is substantially lower at PP than at MF synapses.

In summary, excitatory synapses formed by the PP and MFs onto PIIs have different complements of glutamate receptors: PP synapses express nonrectifying, PhTx-433insensitive, CI-AMPARs and low levels of NMDARs. In contrast, MF synapses contain inwardly rectifying, $\mathrm{PhTx}$-433sensitive, CP-AMPA and higher levels of NMDARs. absence of LTP at the two pathways was related to the expression of $\mathrm{Ca}^{2+}$-permeable $(\mathrm{CP})$ - and $\mathrm{Ca}^{2+}$-impermeable (CI)-AMPARs or NMDARs (Tóth and McBain, 1998; Tóth et al., 2000; Kullmann and Lamsa, 2007; Lamsa et al., 2007; Galván et al., 2008).

First, we tested the presence of CP-AMPARs (Fig. 4) by examining the current-voltage $(I-V)$ relationship of isolated AMPARmediated EPSCs evoked by stimulation of the two pathways (see Materials and Methods). PIIs showed a strong inwardly rectifying $I-V$ relationship of EPSCs evoked at MF synapses (Fig. $4 A$ ). The rectification index $(\mathrm{RI})$ was $0.11 \pm 0.02$ (EPSCs at $+60 \mathrm{mV} /-60$ $\mathrm{mV}$; six cells) (Fig. 4C), consistent with the abundant presence of CP-AMPARs at these synapses (Geiger et al., 1995). In contrast, synaptically evoked EPSCs at PP inputs displayed a linear $I-V$ relationship (RI, $0.7 \pm 0.09$; nine cells), indicating the expression of postsynaptic CI-AMPARs at PP-PII synapses.

To verify these results, we tested the effect of the polyamine toxin philanthotoxin-433 (PhTx-433), a selective blocker of CP-AMPARs lacking the GluR2 subunit (Tóth and McBain, 1998). In agreement with the above results, PhTx-433 blocked EPSC peak amplitudes by $66.6 \pm 7.1 \%$ at MF synapses (four cells; $p=0.029$ ), but had no effect on PP-induced EPSCs (three cells) (Fig. 4B,C). Thus, transmission at MF-PII terminals is primarily mediated by CP-AMPARs, whereas signaling at PP-PII synapses involves CI-AMPARs.

It has been previously shown that glutamatergic inputs onto interneurons containing CP-AMPARs display smaller NMDAR-mediated components than synapses containing CIAMPARs (Angulo et al., 1999; Lei and McBain, 2002; Laezza and Dingledine, 2004; Lamsa et al., 2007). Does this principle apply to DG PIIs? We addressed this question by examining the $I-V$ relationship of NMDAR-mediated components (Fig. 5) in the presence of $20 \mu \mathrm{M} \mathrm{CNQX}$, which resulted in a complete block (>95\%) of

\section{LTP in perisomatic inhibitory interneurons is NMDA} receptor-independent but requires AMPA receptors

At most pyramidal cell inputs, LTP depends on the activation of NMDARs (Bliss and Lomo, 1973; Herron et al., 1986; Kauer et al., 1988) (but see Nicoll and Schmitz, 2005). Thus, the presence of NMDARs at MF-PII synapses raises the question whether they underlie LTP induction (Fig. 6). We addressed this question by applying a BFS to MF inputs paired with depolarizations in the postsynaptic PII in the presence of the NMDAR antagonist D-APV. In all PIIs tested, robust LTP was induced (175.8 \pm 27.8 ; five cells) (Fig. $6 \mathrm{~A}$ ). In fact, the level of potentiation was comparable with that observed in experiments without D-APV (166.4 \pm $25.7 \%$; 10 cells; $p=0.241$ ) (Fig. $3 B, D$ ), indicating that induction and expression of LTP at MF-PII synapses does not depend on NMDAR-mediated transmission.

Next, we tested the involvement of AMPARs in the induction of MF-PII LTP (Fig. 6B). We recorded EPSCs evoked by two independent MF inputs in PIIs. After establishing a stable baseline, $10 \mu \mathrm{M}$ CNQX was bath-applied. This resulted in a marked decrease in the peak amplitude of EPSCs at both inputs (test input, $14.2 \pm 3.1 \%$, and control input, $18.3 \pm 4.6 \%$ remaining EPSC peak amplitude; eight cells) (Fig. 6B). Then, a BFS was applied to one of the two inputs (Fig. 6B, red open circles), and washout of CNQX was immediately started after the induction protocol was delivered. The recovery of MF-mediated EPSCs was monitored for up to $30 \mathrm{~min}$. Peak amplitudes recovered with a similar time course to comparable levels (test input, $80.5 \pm 6.4 \%$, and control input, $82.9 \pm 5.3 \%$; $p=$ 0.175 ) for the paired and control input, respectively, indicating that AMPAR block prevents LTP induction at the tested MF-PII synapses. 
Hebbian LTP persists in PIIs with intact internal milieu

Recently, a novel anti-Hebbian form of synaptic plasticity was identified at excitatory synapses onto CA1 interneurons (Kullmann and Lamsa, 2007; Lamsa et al., 2007). This form of plasticity depends on the unique inwardly rectifying properties of CP-AMPARs produced by intracellular polyamines (Bowie and Mayer, 1995; Koh et al., 1995). Whole-cell recordings may lead to washout of intracellular polyamines and may turn anti-Hebbian plasticity to a Hebbian type. We therefore asked whether Hebbian plasticity observed in our experiments at MF synapses persists in PIIs with intact intracellular milieu (Fig. 7).

To address this question, we performed long-duration ( $\geq 25 \mathrm{~min}$ ) gramicidin perforated-patch recordings from fast-spiking interneurons (adaptation ratio, $1.03 \pm 0.06)$ under current-clamp conditions (Fig. 7A). Application of a BFS to MF inputs paired with depolarization in PIIs resulted in the induction of LTP (Fig. $7 B$ ). The magnitude of the potentiation ( $140.4 \pm 21.5 \%$; 4 cells) was comparable with that observed during normal whole-cell recordings ( $166.4 \pm 25.7 \% ; 10$ cells; $p=0.461$ ) (Figs. $3 B, D, 6 A$ ). Thus, Hebbian MF-LTP persists with unperturbed polyamine content and intracellular milieu in DG PIIs.
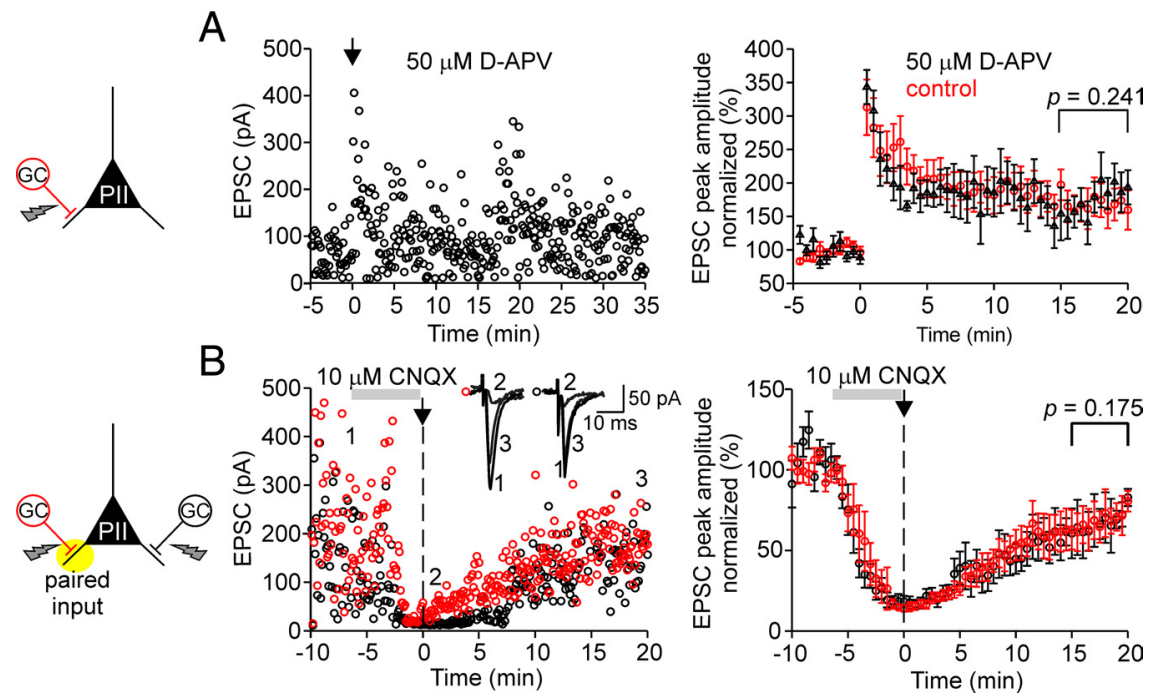

Figure 6. LTP at mossy fiber-perisomatic inhibitory interneuron synapses is NMDA receptor independent but requires AMPA receptor activation. $\boldsymbol{A}$, Left, Individual peak amplitudes of AMPAR-mediated EPSC elicited at MF-PII synapses in the presence of the NMDAR blocker D-APV plotted as a function of time before and after pairing for a single PII. The arrow indicates the time of BFS application. Right, LTP in the presence of D-APV (50 $\mu \mathrm{m}$; black circles) is expressed to the same extent (175.8 $\pm 27.8 \%$ at $15-20$ min after pairing; 5 cells) as in the absence of D-APV (red circles; $166.4 \pm 25.7 \% ; p=0.241$; same data as in Fig. 3B), demonstrating that LTP at MF-PII synapses is not NMDAR dependent. $\boldsymbol{B}$, Block of AMPARs at MF-PII synapses prevents LTP. Left, Amplitudes of individual EPSCs are plotted as a function of time for two independent MF inputs to a single PII. After a baseline period, $10 \mu \mathrm{M}$ CNQX was washed in to block AMPARs ( $V_{\text {hold }}=-70 \mathrm{mV}$; amplitude reduction, $\sim 85 \%$ ). A BFS was applied to one MF input (red circles) at $t=0$ (arrow). Washout of CNQX was started immediately after the pairing protocol. Time course of recovery of the paired MF input (red circles) and the unpaired MF input (black circles) was similar indicating that LTP was not induced. Inset, Average EPSCs during the control period (1), first $30 \mathrm{~s}$ after the pairing (2), and at late phases after LTP induction (15-20 min). Right, Summary of the experimental data from eight cells. Each circle represents the average of EPSCs over 30 s intervals for the paired (black) and control MF input (red) normalized to the mean baseline amplitudes. The gray bars indicate the duration of the CNQX wash-in; the vertical dashed lines refer to the time of the BFS. $\boldsymbol{A}$, Two-tailed Student's $t$ test. $\boldsymbol{B}$, Wilcoxon's signed rank test. Average measurements represent mean \pm SEM.

\section{Discussion}

Our results demonstrate that temporally precise recruitment of fast-spiking PIIs depends on the contiguous activation of the PP and the MF pathway in the DG (Fig. 8). Repeated pairing of the two inputs leads to associative LTP at MF-PII synapses. Induction of MF-PII LTP requires the generation of action potentials in postsynaptic PIIs, which was maximal if PP and MF inputs were paired with a disynaptic latency of $10 \mathrm{~ms}$. This associative plasticity provides a new mechanism by which recruitment of PIIs and thereby feedback inhibition can be dynamically modulated, an important requirement for encoding of information in sparse neuronal assemblies (Leutgeb et al., 2007; Klausberger and Somogyi, 2008; de Almeida et al., 2009).

\section{Recurrent excitation by MFs promotes temporally precise activation of PIIs}

Fast-spiking PIIs such as basket cells are thought to act as rapid signaling devices and produce precisely timed inhibition in their postsynaptic targets (Geiger et al., 1997; Jonas et al., 2004; Doischer et al., 2008). Indeed, powerful, short-latency inhibition by PIIs controls activation of principal cells in response to afferent inputs in the CA1 area (Pouille and Scanziani, 2001). However, certain cortical afferents, such as the PP, terminate on distal dendrites and their postsynaptic effects show considerable attenuation and deceleration at the soma (Nörenberg et al., 2010). Our results demonstrate that, in contrast to proximally located MF synapses (Geiger et al., 1997), distal PP inputs activate DG PIIs with long latency and low precision. Thus, slow feedforward excitation by electrotonically distant synapses alone cannot pro- duce precisely timed perisomatic inhibition in the DG network. However, if a strong PP input is associated with a weak MF input arriving with a short delay, PIIs are discharged efficiently with improved temporal precision. In this scenario, slow PP-mediated EPSPs favor temporal summation, whereas rapid MF-EPSPs define a narrow time window for action potential initiation.

In the intact network, convergence of monosynaptic PP- and disynaptic MF-mediated excitation onto PIIs occurs when the PP input recruits GCs (Leutgeb et al., 2007). Indeed, our data demonstrate that a moderate increase in the activation of the PP elicits disynaptic MF-mediated EPSCs after the monosynaptic PP-EPSCs in PIIs. Interestingly, the optimal delay between the PP and MF input $(\sim 10 \mathrm{~ms})$ for the efficient activation of PIIs and the induction of LTP corresponds well to the latency between the monosynaptic and disynaptic EPSCs. Thus, integration of convergent inputs and induction of associative LTP in PIIs appears to be optimized for network structure in the DG.

\section{Associative plasticity at MF-PII synapses}

MF-PII synapses have been previously shown to express homosynaptic LTP (Alle et al., 2001). The present results further reveal that plasticity at these synapses is promoted by $\mathrm{PP}$ inputs in an associative manner. Conditions for the induction of MF-LTP appear to be similar to the associative, heterosynaptic plasticity induced by the coactivation of PP and Schaffer collateral inputs in CA1 pyramidal cells (Dudman et al., 2007). However, MF-LTP requires action potentials in the postsynaptic PII, whereas heterosynaptic plasticity in principal cells develops in the subthreshold regimen (Dudman et al., 2007), conceivably reflecting 
A
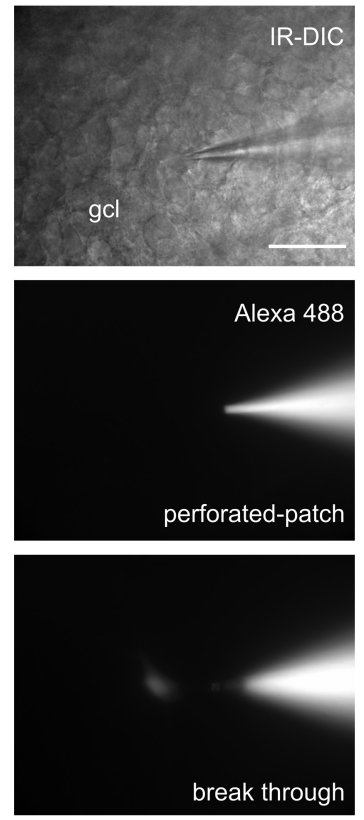

B

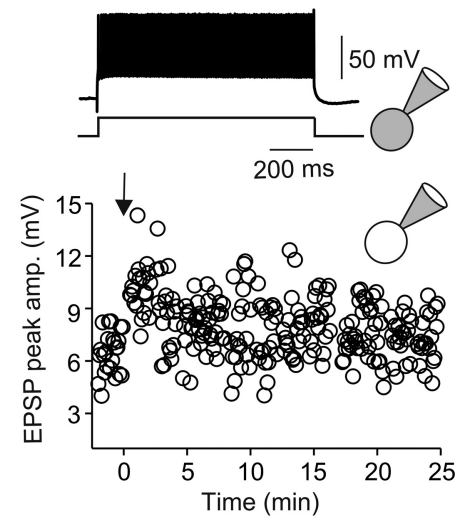

C

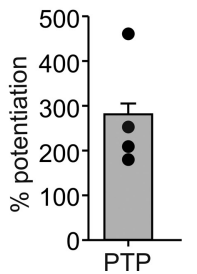

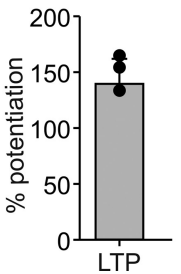

Figure 7. Gramicidin perforated-patch recordings reveal that Hebbian associative LTP persist at mossy fiber-perisomatic inhibitory interneuron synapses with intact internal milieu. $\boldsymbol{A}$, Top, IR-DIC image of a perforated-patch recording from a fast-spiking ( $\boldsymbol{B}$, inset) PII with soma in the gcl. Middle, Epifluorescence image of the same neuron during recording to monitor stability of the perforated patch. Alexa Fluor $488(50 \mu \mathrm{m})$ was added to the pipette solution. Note that the fluorescence signal is restricted to the pipette indicating integrity of the perforated-patch. Bottom image, Spontaneous breakthrough is indicated by labeling of the cell body. $\boldsymbol{B}$, A BFS protocol applied to MF input in association with depolarization in the postsynaptic PII (see Materials and Methods) at $t=0 \mathrm{~ms}$ (arrow) evokes stable LTP in the perforated-patch configuration. EPSP peak amplitude is plotted against time before and after the BFS. Inset on top, At the end of the LTP recording, the same cell was repatched to show its fast-spiking, nonadapting action potential phenotype (adaptation ratio, 0.81) during long-lasting depolarizing current injections ( $600 \mathrm{pA} ; 1 \mathrm{~s})$. C, Comparison of PTP and LTP in four perforated-patch recorded Plls. The bars represent mean \pm SEM. The circles represent single data points.

differences in the electrotonic distance of the involved synapses as well as in molecular mechanisms (see below). Recently, however, a spike time-dependent form of plasticity was also described in CA1 pyramidal cells, which was modulated by the relative timing of PP and Schaffer collateral inputs (Kwag and Paulsen, 2009).

The dependence of MF-LTP on action potential generation reflects a form of Hebbian plasticity (Hebb, 1949; Caporale and Dan, 2008) in DG PIIs, a finding that contrasts the anti-Hebbian plasticity observed in the CA1 feedback inhibitory circuit (Lamsa et al., 2007). We propose that differences in cellular and synaptic properties and network architecture define distinct conditions for synaptic plasticity and that these conditions can vary among brain regions (Sjöström and Häusser, 2006; Lamsa et al., 2007; Takahashi and Magee, 2009).

\section{Molecular mechanisms of synapse-specific plasticity}

What are the molecular mechanisms underlying the differential expression of LTP at MF-PII and PP-PII synapses? Differences in the pharmacological properties and $I-V$ relationships of MF- and PP-mediated signals suggest that the two major PII inputs express distinct complements of postsynaptic glutamate receptors with different $\mathrm{Ca}^{2+}$ signaling mechanisms. The associative nature of LTP would be consistent with NMDAR-mediated coincidence detection (Bliss and Collingridge, 1993; Lamsa et al., 2005; Dudman et al., 2007). Surprisingly, blocking NMDARs did not

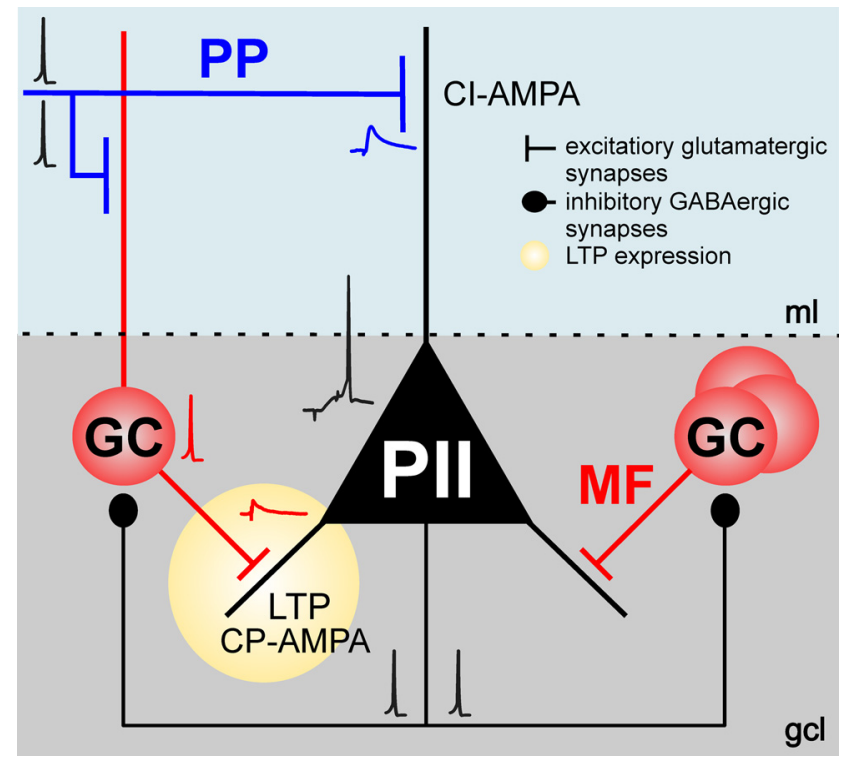

Figure 8. Schematic illustration of the mechanisms underlying pathway-specific LTP at an excitatory synapse onto perisomatic inhibitory interneurons. Repeated coactivation of PP and MF inputs converging onto a postsynaptic PII with a disynaptic latency of $\sim 10$ ms induces LTP at MF synapses. LTP requires CP-AMPARs that are selectively expressed at MF-PII terminals. In contrast, PP-PII synapses express predominantly CI-AMPARs and do not show LTP in our experiments. Synaptic plasticity at MF-PII synapses leads to enhanced recruitment of Plls and, thereby, an increased feedback inhibition in the DG circuitry, an important requirement for maintaining sparse activity in the principal cell population and a high signal-to-noise ratio in information processing. See text for additional details. The traces represent average EPSPs and single action potentials.

prevent MF-LTP induction, indicating that they are not essential for this form of plasticity. In contrast, LTP induction is dependent on AMPAR activation (Fig. 6) and shows good correlation with the expression of CP-AMPARs at MF-PII synapses. Therefore, $\mathrm{Ca}^{2+}$ influx via CP-AMPARs (Geiger et al., 1995) could serve as trigger mechanism for MF-LTP. Consistently, LTP induction at this synapse has been shown to require postsynaptic $\mathrm{Ca}^{2+}$ signaling (Alle et al., 2001). In contrast, LTP expression appears to be presynaptic, implying some form of retrograde signaling (Alle et al., 2001).

$\mathrm{Ca}^{2+}$ permeability of AMPARs depends on the presence or absence of GluR2 subunits (Geiger et al., 1995; Tóth and McBain, 1998; Topolnik et al., 2005; Isaac et al., 2007). Receptors lacking GluR2 show high permeability for $\mathrm{Ca}^{2+}$ and display inward rectification because of a block by cytoplasmic polyamines at depolarized membrane potentials (Jonas et al., 1994; Isaac et al., 2007; Lamsa et al., 2007; Kullmann and Lamsa, 2008). Thus, rectification by CP-AMPARs is opposite to that by NMDARs, and they have been shown to underlie anti-Hebbian rather than Hebbian plasticity in CA1 interneurons (Lamsa et al., 2007; Kullmann and Lamsa, 2008). However, there is evidence that CP-AMPARs contribute to tetanus-induced LTP in interneurons of the amygdala (Mahanty and Sah, 1998). Interestingly, repetitive activation can temporarily relieve the polyamine block and enhance CPAMPARs conductance (Rozov and Burnashev, 1999; Tóth et al., 2000). This mechanism conceivably plays a role in the burst stimulation paradigm used in our experiments and under physiological conditions when highly excited GCs discharge in bursts (Jung and McNaughton, 1993).

Although a CP-AMPAR-mediated mechanism may adequately explain the induction of MF-LTP, the dependence of LTP on postsynaptic action potential generation suggests that addi- 
tional $\mathrm{Ca}^{2+}$ sources may be involved. Basket cells express voltagedependent $\mathrm{Ca}^{2+}$ channels (VDCCs) on their soma and proximal dendrites (Aponte et al., 2008; Bucurenciu et al., 2008). $\mathrm{Ca}^{2+}$ entry through these channels during action potentials could support LTP induction at proximal MF inputs (Galván et al., 2008; Topolnik et al., 2009). Furthermore, group I mGluRs may contribute to $\mathrm{Ca}^{2+}$ entry into interneurons, boosted by the strong postsynaptic depolarization such as during action potentials (Topolnik et al., 2005). Thus, $\mathrm{Ca}^{2+}$ transients mediated by VDCCs, CP-AMPA, and mGluRI receptors may act synergistically to induce LTP at MF-PII synapses (Topolnik et al., 2005, 2009). Conversely, the apparent absence of synaptic plasticity at $\mathrm{PP}$ inputs in our experiments could be explained by the absence of CP-AMPARs, the negligible NMDAR-mediated component (present results) and the small voltage-dependent $\mathrm{Ca}^{2+}$ transients in distal dendrites of PIIs (Aponte et al., 2008).

Compared with the properties of MF-PII LTP observed in our study, plasticity of MF synapses on CA3 interneurons shows several differences but also some similarities. Most prominently, only long-term depression (LTD) was found at MF-stratum lucidum interneuron (SLIN) synapses in naive slices (Tóth et al., 2000; Lei and McBain, 2002, 2004). MF-SLIN synapses express both CI- and CP-AMPARs (Tóth et al., 2000; Lei and McBain, 2002). However, only synapses containing CP-AMPARs can switch from LTD to LTP after internalization of presynaptic mGluR7 (Pelkey et al., 2005). There are also differences in LTP induction: whereas in MF-PII synapses it depends on postsynaptic depolarization, MF-SLIN-mediated LTP emerges at resting potential. In stratum lacunosum-moleculare interneurons (SM), a Hebbian form of LTP was observed requiring strong depolarization during high-frequency stimulation (Galván et al., 2008). Similar to MF-PII and MF-SLIN synapses, LTP at MF-SM inputs is independent of NMDARs (Pelkey et al., 2005) and requires CI-AMPARs (Galván et al., 2008).

\section{Function of synaptic plasticity in PIIs for information processing}

What is the functional role of plasticity at MF-PII synapses for information processing in the DG network? We show that excitatory MF inputs are crucial for the temporally precise recruitment of PIIs. This input can derive from a small subset of highly excited GCs during activation by the entorhinal cortex (Leutgeb et al., 2007). The rapid and powerful inhibition mediated by recruited PIIs counterbalances afferent excitation and prevents additional activation in the network. Suppression of less excited cells, after strongly excited cells have discharged, leads to a "winner-takes-all" situation and maintains sparse activity in the network (de Almeida et al., 2009).

Plasticity of MF-mediated excitation provides a new level of integration in this microcircuit enabling a dynamic recruitment of PIIs. Potentiation of MF synapses conceivably occurs in PIIs postsynaptic to GCs that are activated repeatedly by PP inputs and themselves subject to plastic changes at their input. As a consequence, functional association of GCs and a selected population of PIIs will develop. The enhanced activation of PIIs will increase the signal-to-noise ratio during assembly patterns, promote pattern separation, and thus can enhance storage capacity in the network (Hafting et al., 2005; Fyhn et al., 2007; Leutgeb et al., 2007; McHugh et al., 2007).

\section{References}

Acsády L, Kamondi A, Sík A, Freund T, Buzsáki G (1998) GABAergic cells are the major postsynaptic targets of mossy fibers in the rat hippocampus. J Neurosci 18:3386-3403.
Alle H, Jonas P, Geiger JR (2001) PTP and LTP at a hippocampal mossy fiber-interneuron synapse. Proc Natl Acad Sci U S A 98:14708-14713.

Anderson P, Bliss TV, Skrede KK (1971) Lamellar organization of hippocampal excitatory pathways. Exp Brain Res 13:222-234.

Angulo MC, Rossier J, Audinat E (1999) Postsynaptic glutamate receptors and integrative properties of fast-spiking interneurons in the rat neocortex. J Neurophysiol 82:1295-12302.

Aponte Y, Bischofberger J, Jonas P (2008) Efficient $\mathrm{Ca}^{2+}$ buffering in fastspiking basket cells of rat hippocampus. J Physiol 586:2061-2075.

Bartos M, Vida I, Frotscher M, Geiger JR, Jonas P (2001) Rapid signaling at inhibitory synapses in a dentate gyrus interneuron network. J Neurosci 21:2687-2698.

Bartos M, Vida I, Frotscher M, Meyer A, Monyer H, Geiger JR, Jonas P (2002) Fast synaptic inhibition promotes synchronized gamma oscillations in hippocampal interneuron networks. Proc Natl Acad Sci U S A 99:13222-13227.

Bartos M, Vida I, Jonas P (2007) Synaptic mechanisms of synchronized gamma oscillations in inhibitory interneuron networks. Nat Rev Neurosci 8:45-56.

Bliss TV, Collingridge GL (1993) A synaptic model of memory: long-term potentiation in the hippocampus. Nature 361:31-39.

Bliss TV, Lomo T (1973) Long-lasting potentiation of synaptic transmission in the dentate area of the anaesthetized rabbit following stimulation of the perforant path. J Physiol 232:331-356.

Bowie D, Mayer ML (1995) Inward rectification of both AMPA and kainate subtype glutamate receptors generated by polyaminemediated ion channel block. Neuron 15:453-462.

Bragin A, Jandó G, Nádasdy Z, Hetke J, Wise K, Buzsáki G (1995) Gamma $(40-100 \mathrm{~Hz})$ oscillation in the hippocampus of the behaving rat. J Neurosci 15:47-60.

Bucurenciu I, Kulik A, Schwaller B, Frotscher M, Jonas P (2008) Nanodomain coupling between $\mathrm{Ca}^{2+}$ channels and $\mathrm{Ca}^{2+}$ sensors promotes fast and efficient transmitter release at a cortical GABAergic synapse. Neuron 57:536-545.

Buzsáki G (2002) Theta oscillations in the hippocampus. Neuron 33: 325-340.

Caporale N, Dan Y (2008) Spike timing-dependent plasticity: a Hebbian learning rule. Annu Rev Neurosci 31:25-46.

Davis S, Butcher SP, Morris RG (1992) The NMDA receptor antagonist D-2-amino-5-phosphonopentanoate (D-AP5) impairs spatial learning and LTP in vivo at intracerebral concentrations comparable to those that block LTP in vitro. J Neurosci 12:21-34.

de Almeida L, Idiart M, Lisman JE (2009) A second function of gamma frequency oscillations: an E\%-max winner-take-all mechanism selects which cells fire. J Neurosci 29:7497-7503.

Dietrich D, Beck H, Kral T, Clusmann H, Elger CE, Schramm J (1997) Metabotropic glutamate receptors modulate synaptic transmission in the perforant path: pharmacology and localization of two distinct receptors. Brain Res 767:220-227.

Doischer D, Hosp JA, Yanagawa Y, Obata K, Jonas P, Vida I, Bartos M (2008) Postnatal differentiation of basket cells from slow to fast signaling devices. J Neurosci 28:12956-12968.

Dudman JT, Tsay D, Siegelbaum SA (2007) A role of synaptic inputs at distal dendrites: instructive signals for hippocampal long-term plasticity. Neuron 56:866-879.

Freund TF, Buzsáki G (1996) Interneurons of the hippocampus. Hippocampus 6:347-470.

Fyhn M, Hafting T, Treves A, Moser MB, Moser EI (2007) Hippocampal remapping and grid realignment in entorhinal cortex. Nature 446:190-194.

Galván EJ, Calixto E, Barrionuevo G (2008) Bidirectional Hebbian plasticity at hippocampal mossy fibre synapses on CA3 interneurons. J Neurosci 24:14042-14055.

Geiger JR, Melcher T, Koh DS, Sakmann B, Seeburg PH, Jonas P, Monyer H (1995) Relative abundance of subunit mRNAs determines gating and $\mathrm{Ca}^{2+}$ permeability of AMPA receptors in principal neurons and interneurons in rat CNS. Neuron 15:193-204.

Geiger JR, Lübke J, Roth A, Frotscher M, Jonas P (1997) Submillisecond AMPA receptor-mediated signaling at a principal neuron-interneuron synapse. Neuron 18:1009-1023.

Hafting T, Fyhn M, Molden S, Moser MB, Moser EI (2005) Microstructure of a spatial map in the entorhinal cortex. Nature 436:801-806. 
Häusser M, Roth A (1997) Estimating the time course of the excitatory synaptic conductance in neocortical pyramidal cells using a novel voltage jump method. J Neurosci 17:7606-7625.

Hebb DO (1949) The organization of behavior. New York: Wiley.

Herron CE, Lester RA, Coan EJ, Collingridge GL (1986) Frequencydependent involvement of NMDA receptors in the hippocampus: a novel synaptic mechanism. Nature 322:265-268.

Isaac JT, Ashby M, McBain CJ (2007) The role of the GluR2 subunit in AMPA receptor function and synaptic plasticity. Neuron 54:859-871.

Jonas P, Racca C, Sakmann B, Seeburg PH, Monyer H (1994) Differences in $\mathrm{Ca}^{2+}$ permeability of AMPA-type glutamate receptor channels in neocortical neurons caused by differential GluR-B subunit expression. Neuron 12:1281-1289.

Jonas P, Bischofberger J, Fricker D, Miles R (2004) Interneuron diversity series: fast in, fast out-temporal and spatial signal processing in hippocampal interneurons. Trends Neurosci 27:30-40.

Judge SJ, Hasselmo ME (2004) Theta rhythmic stimulation of stratum lacunosum-moleculare in rat hippocampus contributes to associative LTP at a phase offset in stratum radiatum. J Neurophysiol 92:1615-1624.

Jung MW, McNaughton BL (1993) Spatial selectivity of unit activity in the hippocampal granular layer. Hippocampus 3:165-182.

Kauer JA, Malenka RC, Nicoll RA (1988) NMDA application potentiates synaptic transmission in the hippocampus. Nature 21:250-252.

Klausberger T, Somogyi P (2008) Neuronal diversity and temporal dynamics: the unity of hippocampal circuit operations. Science 321:53-57.

Koh DS, Burnashev N, Jonas P (1995) Block of native $\mathrm{Ca}^{2+}$-permeable AMPA receptors in rat brain by intracellular polyamines generates double rectification. J Physiol 486:305-312.

Kullmann DM, Lamsa KP (2007) Long-term synaptic plasticity in hippocampal interneurons. Nat Rev Neurosci 8:687-699.

Kullmann DM, Lamsa K (2008) Roles of distinct glutamate receptors in induction of anti-Hebbian long-term potentiation. J Physiol 586:1481-1486.

Kwag J, Paulsen O (2009) The timing of external input controls the sign of plasticity at local synapses. Nat Neurosci 12:1219-1221.

Laezza F, Dingledine R (2004) Voltage-controlled plasticity at GluR2deficient synapses onto hippocampal interneurons. J Neurophysiol 92:3575-3581.

Lamsa K, Heeroma JH, Kullmann DM (2005) Hebbian LTP in feed-forward inhibitory interneurons and the temporal fidelity of input discrimination. Nat Neurosci 8:916-924.

Lamsa KP, Heeroma JH, Somogyi P, Rusakov DA, Kullmann DM (2007) Anti-Hebbian long-term potentiation in the hippocampal feedback inhibitory circuit. Science 315:1262-1266.

Lei S, McBain CJ (2002) Distinct NMDA receptors provide differential modes of transmission at mossy fiber-interneuron synapses. Neuron 33:921-933.

Lei S, McBain CJ (2004) Two loci of expression for long-term depression at hippocampal mossy fiber-interneuron synapses. J Neurosci 24:2112-2121.

Leutgeb JK, Leutgeb S, Moser MB, Moser EI (2007) Pattern separation in the dentate gyrus and CA3 of the hippocampus. Science 315:961-966.

Macek TA, Winder DG, Gereau RW 4th, Ladd CO, Conn PJ (1996) Differential involvement of group II and group III mGluRs as autoreceptors at lateral and medial perforant path synapses. J Neurophysiol 76:3798-3806.

Mahanty NK, Sah P (1998) Calcium-permeable AMPA receptors mediate long-term potentiation in interneurons in the amygdala. Nature 394: 683-687.

Major G, Evans JD, Jack JJ (1993) Solutions for transients in arbitrarily branching cables: II. Voltage clamp theory. Biophys J 65:450-468.
McHugh TJ, Jones MW, Quinn JJ, Balthasar N, Coppari R, Elmquist JK Lowell BB, Fanselow MS, Wilson MA, Tonegawa S (2007) Dentate gyrus NMDA receptors mediate rapid pattern separation in the hippocampal network. Science 317:94-99.

Nicoll RA, Schmitz D (2005) Synaptic plasticity at hippocampal mossy fibre synapses. Nat Rev Neurosci 6:863-876.

Nicoll RA, Kauer JA, Malenka RC (1988) The current excitement in longterm potentiation. Neuron 1:97-103.

Nitz D, McNaughton B (2004) Differential modulation of CA1 and dentate gyrus interneurons during exploration of novel environments. J Neurophysiol 91:863-872.

Nörenberg A, Hu H, Vida I, Bartos M, Jonas P (2010) Non-uniform cable properties optimize rapid signaling in fast-spiking GABAergic interneurons. Proc Natl Acad Sci U S A 117:894-899.

Paulsen O, Moser EI (1998) A model of hippocampal memory encoding and retrieval: GABAergic control of synaptic plasticity. Trends Neurosci 21:273-278.

Pelkey KA, Lavezzari G, Racca C, Roche KW, McBain CJ (2005) mGluR7 is a metaplastic switch controlling bidirectional plasticity of feedforward inhibition. Neuron 46:89-102.

Pouille F, Scanziani M (2001) Enforcement of temporal fidelity in pyramidal cells by somatic feed-forward inhibition. Science 293:1159-1163.

Rall W, Segev I (1985) Space-clamp problems when voltage clamping branched neurons with intracellular microelectrodes. In: Voltage and patch clamping with microelectrodes (Smith TG Jr, Lecar H, Redman SJ, Gage P, eds), pp 191-215. Bethesda, MD: American Physiological Society.

Rozov A, Burnashev N (1999) Polyamine-dependent facilitation of postsynaptic AMPA receptors counteracts paired-pulse depression. $\mathrm{Na}$ ture 401:594-598.

Sjöström PJ, Häusser M (2006) Cooperative switch determines the sign of synaptic plasticity in distal dendrites of neocortical pyramidal neurons. Neuron 51:227-238.

Takahashi H, Magee JC (2009) Pathway interactions and synaptic plasticity in the dendritic tuft regions of CAl pyramidal neurons. Neuron 62:102-111.

Topolnik L, Congar P, Lacaille JC (2005) Differential regulation of metabotropic glutamate receptor- and AMPA receptor-mediated dendritic $\mathrm{Ca}^{2+}$ signals by presynaptic and postsynaptic activity in hippocampal interneurons. J Neurosci 25:990-1001.

Topolnik L, Chamberland S, Pelletier JG, Ran I, Lacaille JC (2009) Activitydependent compartmentalized regulation of dendritic $\mathrm{Ca}^{2+}$ signaling in hippocampal interneurons. J Neurosci 29:4658-4663.

Tóth K, McBain CJ (1998) Afferent-specific innervation of two distinct AMPA receptor subtypes on single hippocampal interneurons. Nat Neurosci 1:572-578.

Tóth K, Suares G, Lawrence JJ, Philips-Tansey E, McBain CJ (2000) Differential mechanisms of transmission at three types of mossy fiber synapse. J Neurosci 20:8279-8289.

Treves A, Rolls ET (1994) Computational analysis of the role of the hippocampus in memory. Hippocampus 4:374-391.

Tukker JJ, Fuentealba P, Hartwich K, Somogyi P, Klausberger T (2007) Cell type-specific tuning of hippocampal interneuron firing during gamma oscillations in vivo. J Neurosci 27:8184-8189.

Zipp F, Nitsch R, Soriano E, Frotscher M (1989) Entorhinal fibers form synaptic contacts on parvalbumin-immunoreactive neurons in the rat fascia dentata. Brain Res 495:161-166. 